\title{
Insight into the Significance of Hall Current and Joule Heating on the Dynamics of Darcy-Forchheimer Peristaltic Flow of Rabinowitsch Fluid
}

\author{
Ji-Huan He ${ }^{10}{ }^{1,2,3}$ and Doaa R. Mostapha ${ }^{4}{ }^{4}$ \\ ${ }^{1}$ School of Science, Xi'an University of Architecture and Technology, Xi'an, China \\ ${ }^{2}$ School of Mathematics and Information Science, Henan Polytechnic University, Jiaozuo, China \\ ${ }^{3}$ National Engineering Laboratory for Modern Silk, College of Textile and Clothing Engineering, Soochow University, \\ 199 Ren-Ai Road, Suzhou, China \\ ${ }^{4}$ Department of Mathematics, Faculty of Education, Ain Shams University, Roxy, Cairo, Egypt
}

Correspondence should be addressed to Ji-Huan He; hejihuan@suda.edu.cn

Received 9 August 2021; Accepted 23 September 2021; Published 12 October 2021

Academic Editor: M. M. Bhatti

Copyright (c) 2021 Ji-Huan He and Doaa R. Mostapha. This is an open access article distributed under the Creative Commons Attribution License, which permits unrestricted use, distribution, and reproduction in any medium, provided the original work is properly cited.

\begin{abstract}
This paper aims to present the significance of the Hall current and Joule heating impacts on a peristaltic flow of a Rabinowitsch fluid through tapered tube. The Darcy-Forchheimer scheme is used for a porous medium; a mild stenosis is considered to study the impacts of radiative heat transfer and chemical reactions. Convective conditions are postulated for heat and mass transfer. In the meantime, the slip conditions are presumed for the velocity distribution. Soret and Dufour features bring the coupled differential systems. The hypotheses of a long wavelength and low Reynolds number are employed to approximate the governing equations of motion, and finally the homotopy perturbation method is adopted for numerical study. Pumping characteristics are revealed and the trapping procedure correlated with peristaltic transport is elucidated. The present study is very important in many medical applications, such as the gastric juice motion in the small intestine and the flow of blood in arteries. The mechanism of peristaltic transport with mild stenosis has been exploited for industrial applications like sanitary fluid transport and blood pumps in heart-lung machine. The influences of various physical parameters of the problem are debated and graphically drawn across a set of figures. It is noted that the axial velocity is reduced with the increase of the Hartmann number. However, enhancing both the Rabinowitsch parameter and the Forchheimer parameter gives rise to the fluid velocity. As well, it is debated that Rabinowitsch fluid produces a cubic term of pressure gradient. Therefore, the relation between mean flow rate and the pressure rise does not stay linear. It is recognized that the temperature rises with the enhancement of both Dufour number and Soret number. Furthermore, it is illustrated that the concentration impedes with the increase of the mass transfer Biot number. Also, it is revealed that the trapped bolus contracts in size by enlarging the maximum height of stenosis.
\end{abstract}

\section{Introduction}

Non-Newtonian fluids have a major role in the engineering, industrial systems, medical sciences, textile engineering, and materials science as well. Rabinowitsch [1] established a new model in scrutinizing basic properties of the non-Newtonian fluids. Wada and Hayashi [2] characterized the nonlinear rapport between shear stress and shear strain rate by employing the Rabinowitsch fluid in an experiential cubic equation. Singh et al. [3] determined a model of three major categories of fluids counting on different values of a nonlinear factor, and it can depict the behaviour of Newtonian fluids, shear thickening fluids, and shear thinning fluids. The Rabinowitsch model has many major implementations in the analysis of the squeeze film, annular disk, externally pressurized bearing, journal bearing, and pivoted curved slider bearings. The transit of fluid due to the symmetric contraction and expansion of flexible walls is recognized as a 
peristaltic transport, and peristalsis has certainly determined the attention of physiologists, because the foremost mechanism for transporting fluids in many living organisms is the peristaltic transport; for illustration, see Burns and Parkes [4]. Peristalsis has many implementations in physiological processes, such as swallowing of food through esophagus, urine transport from kidney to bladder through the ureter, transport of blood in heart, transport of lymph in the lymphatic vessels, the vasomotion of small blood vessels, motion of spermatozoa in cervical canal, transport of bile in bile duct, and some worms; for instance, see Shapiro et al. [5]. Additionally, it has many promising applications in engineering and industry. These achievements can be involved in the biomedical instruments with the heart-lung machine as well as the pumping of toxic and acidic fluids with the aid of pumps [6]. Shapiro et al. [5] and Fung and Yih [7] scrutinized the peristaltic flows with some hypotheses, such as long wavelength approximation, low Reynolds number, and small amplitude ratio. The problem of peristaltic flow in a tube of Rabinowitsch fluid was scrutinized by Singh and Singh [8]. Singh [9] examined the exact solution of Rabinowitsch peristaltic flow in a tube, and found that the pseudoplastic and dilatant fluids counted on the instantaneous flow rate.

One of the fundamental topics of scientific research is the circulatory disorders. The cardiovascular diseases, such as stenosis or arteriosclerosis, are concerned with the flow conditions in the blood vessels. Therefore, the scrutiny of blood flow across stenosed arteries is very serious. Stenosis can be recognized as narrowing of any body passage [10]. It can be displayed in many diseases such as cerebral strokes, myocardial infarction, and heart failure by reducing or occluding the blood supply [11]. Moreover, in case of stenosed arterial, stresses and resistance flow are more elevated than those in the case of the normal ones. Verma and Parihar [12] investigated the streaming of blood flow cross a tapered artery with mild stenosis and hematocrit. El-dabe et al. [13] discussed the electrohydrodynamic (EHD) peristaltic flow of a viscoelastic Oldroyd fluid in the presence of mild stenosis cross an endoscope. They found that the rise in the maximum height of stenosis diminishes the velocity of the flow. Heat transfer has a major aspect in scrutinizing the chemical engineering and industrial implementations. Heat transfer can be found in various implementations such as mobile phones, radioactive treatments, and radiofrequency therapy [14]. Sadaf and Nadeem [15] scrutinized the peristaltic flow of Rabinowitsch fluid model under the act of viscous dissipation and convective boundary conditions. They displayed that when the Biot number enhances, the thermal conductivity is increased, which contributes to reducing temperature. In the recent past, the impacts of heat and mass transfer on biofluids are scrutinized by Ijaz et al. [16]. Recently, the investigation of heat and mass transfer on the blood flow cross stenosed arteries has been scrutinized by Chakravarty and Sen [17]. Furthermore, the investigation of heat and mass transfer via chemical reactions is of broad physical significance in the chemical and hydrometallurgical industries. The impact of radiative heat transfer is very substantial for manufacturing industries, especially in the design of equipment, nuclear plants, and gas turbines. By the same token, the impacts of thermal radiation on forced and free convection flow are very paramount in the content of space technology and processes of high temperature. Pal and Talukdar [18] investigated the combined impacts of chemical reaction and thermal radiation in the presence of viscous dissipating fluid over a vertical plate in porous media. Via the Newtonian fluids, the impacts of thermal diffusion (Soret) and diffusion-thermo (Dufour) may be omitted. On the contrary, it is inconvenient to incorporate these impacts in the case of the non-Newtonian fluids, as shown by Hayat et al. [19]. Shah et al. [20] examined the significance of nanoparticle's radius, heat flux due to concentration gradient, and mass flux due to temperature gradient in the case of water conveying copper nanoparticles. They discussed the influence of heat flux due to concentration gradient, and mass flux due to temperature gradient.

In recent years, the investigation of magnetohydrodynamic (MHD) flow problems has gained increasing interest due to its comprehensive engineering and medical implementations. MHD involves many implementations in the cell separation, the magnetic wound, or the cancer tumor treatment [21-23]. Several theoretical articles have scrutinized the impact of magnetic field on the peristaltic flow as given by Eldabe and Mostapha [24] and Bhatti et al. [25]. Hall current is another major portion of the MHD peristaltic flow. It has major implementations in engineering fields, such as power generators, heating elements, Hall accelerators, and MHD generators [26-29]. In addition, Joule heating happens when the energy of an electric current turns into heat cross resistance. It provides many implementations in biomedical and nuclear engineering, fluid industries, geophysical flows, resistance thermometers, and thermostats [30]. Hayat et al. [31] scrutinized the impacts of Hall current and Joule heating on peristaltic flow under convective boundary conditions. They found that larger values of Hall parameter lead to decreasing the effective conductivity. It causes the decreasing in the magnetic damping force. So, the axial velocity is enhanced. Eldabe and Mostapha [32] scrutinized Hall current and Joule heating impacts on the peristaltic flow of a Sisko fluid. The flow streams across mild stenosis in the presence of a porous medium in a tapered artery having the impacts of slip and convective boundary conditions. They found that the axial velocity enhances with the elevation of Hall current parameter and with the diminution of the Hartmann number. Flow through porous space is involved in modern research because of its applicability in hydrology, soil mechanics, dying wood, and several other usages. Transmission across the porous structure is very fundamental due to the intersection between the column wall and fluid packing particles. The flow under small velocity conditions and low porosity is introduced by Darcy's theory [33]. Forchheimer [34] has upgraded the equation by attaching the square term of velocity in the Darcy equation. Also, Shafiq et al. [35] recognized this term as the Forchheimer term. Eid and Mabood [36] investigated the impacts of Darcy-Forchheimer scheme on the entropy analysis of a hydromagnetic micropolar dusty carbon NTs-kerosene 
nanofluid with heat generation. They found that the rise in the Forchheimer number reduces the velocity. Khan et al. [37] scrutinized Dufour and Soret impacts on Darcy-Forchheimer flow of second-grade fluid with the variable magnetic field and thermal conductivity. They revealed that the Forchheimer number and porosity parameter raise the skin friction coefficient.

In accordance with the aforementioned aspects, the current article pursues to investigate the Darcy-Forchheimer peristaltic Rabinowitsch fluid flow in a tapered tube in the presence of mild stenosis. The impacts of Hall current and Joule heating are taken into consideration. Furthermore, the slip condition of velocity distribution and convective conditions of heat and mass transfer are also hypothesized. Additionally, the impacts of radiation, viscous dissipation, Soret and Dufour effects, and chemical reactions are acknowledged. The governing equations of motion are simplified by employing longer wavelength and low Reynolds number assumptions. These equations are analytically solved with regard to the adequate boundary conditions by utilizing various analytical methods [38-44]. The distributions of an axial velocity, temperature, and concentration are achieved. The pressure rise and friction force are obtained by employing a numerical integration. Numerical calculations are analyzed to yield the impacts of several parameters. To crystallize the presentation of the study, this study is to answer to the following related research questions:

(1) How to establish a differential model for the motion under the adequate boundary conditions

(2) How to use the homotopy perturbation method to solve the problem
(3) How to reveal essentially the solution graphically and how to elucidate the pumping characteristics

\section{Formulation of the Problem}

An unsteady motion of a Rabinowitsch fluid flowing across a tapered tube is investigated. The tube has a sinusoidal wave transmit on its wall in the presence of mild stenosis. The flow goes through a Darcy-Forchheimer porous medium. The system is acted upon by a uniform strong magnetic field of strength $B_{0}$. The Hall and Joule heating impacts are postulated. By contrast, the consequence of the electric field is disregarded. The radiative heat and mass transfer are inspected together with the impacts of viscous dissipation. For more convenience, the cylindrical polar coordinate system $(R, \theta, Z)$ is employed so that the $Z$-axis can coincide with the axis of the tubes. The presence of Hall current produces a force in the $\theta$-direction. Subsequently, the flow occupies the three dimensions. For simplicity, no impacts of heat transfer and concentration properties are presumed in the $\theta$-direction. The tube is convectively heated. Also, the convective mass condition and the slip condition of velocity distribution are presumed. It is supposed that the wall of the tube is heated by a constant temperature $T^{*}$ and concentration $C^{*}$. Heat and mass transfer generate the coefficients $h_{t}$ and $h_{c}$. Chemical reaction and Soret and Dufour impacts are also hypothesized. The magnetic Reynolds number is taken of very small value; it follows that the induced magnetic field is smaller in comparison to the external magnetic field. Therefore, the induced magnetic field can be omitted. The sketch of the problem is modeled in Figure 1.

As given by Verma and Parihar [12], the effective radius of the tapered artery $R(z)$ is taken as follows:

$$
R(z)= \begin{cases}R_{1}-\delta m_{1}(Z+L+d), & -L \leq Z<-Z_{0}, \\ R_{1}-\delta m_{1}(Z+L+d)-\frac{H_{0}}{2}\left[1+\cos \frac{\pi Z}{Z_{0}}\right], & -Z_{0} \leq Z \leq Z_{0}, \\ R_{1}-\delta m_{1}(Z+L+d), & Z_{0}<Z \leq d,\end{cases}
$$

where $R_{1}$ represents the radius of the artery, $\delta=R_{1} / \lambda, \lambda$ is the wavelength, $H_{0}=h \cos \phi$ is the height of the stenosis in the tapered artery, $\phi$ is the angle of tapering, $h$ is the maximum height of the stenosis, $z_{0}$ is the half-length of the stenosis, and $m_{1}=\tan \phi$ is the slope of the tapered artery.

The ratio between the height of the stenosis and the radius of the normal artery is taken to be much less than unity $\left(H_{0} / r\right)$. The artery is presumed to be of finite length $L+d$ [45]. The current paper condenses on all the possible different shapes of the artery, namely, the converging tapering $\phi<0$, nontapered artery $\phi=0$, and the diverging tapering $\phi>0$ [46].

As given by Wada and Hayashi [2], the imposed nonNewtonian fluid is coordinated by the Rabinowitsch model.
It follows that the constitutive equation can be written in the following form:

$$
\mu \underline{\dot{\gamma}}=\underline{\tau}+a \underline{\underline{\tau}}
$$

where

$$
\underline{\dot{\gamma}}=\nabla \underline{V}+(\nabla \underline{V})^{T^{*}},
$$

is the rate of strain tensor, $V=(U, 0, W)$ is the velocity of the fluid, $T^{*}$ represents the matrix transpose, $\mu$ is the initial viscosity, $\underline{\tau}$ is the shear stress in the fluid, and $a$ means the nonlinear factor responsible for the non-Newtonian effects of the fluid. For a uniform value of $\mu$, it follows that the nonlinearity of the flow curve will be enhanced with the 


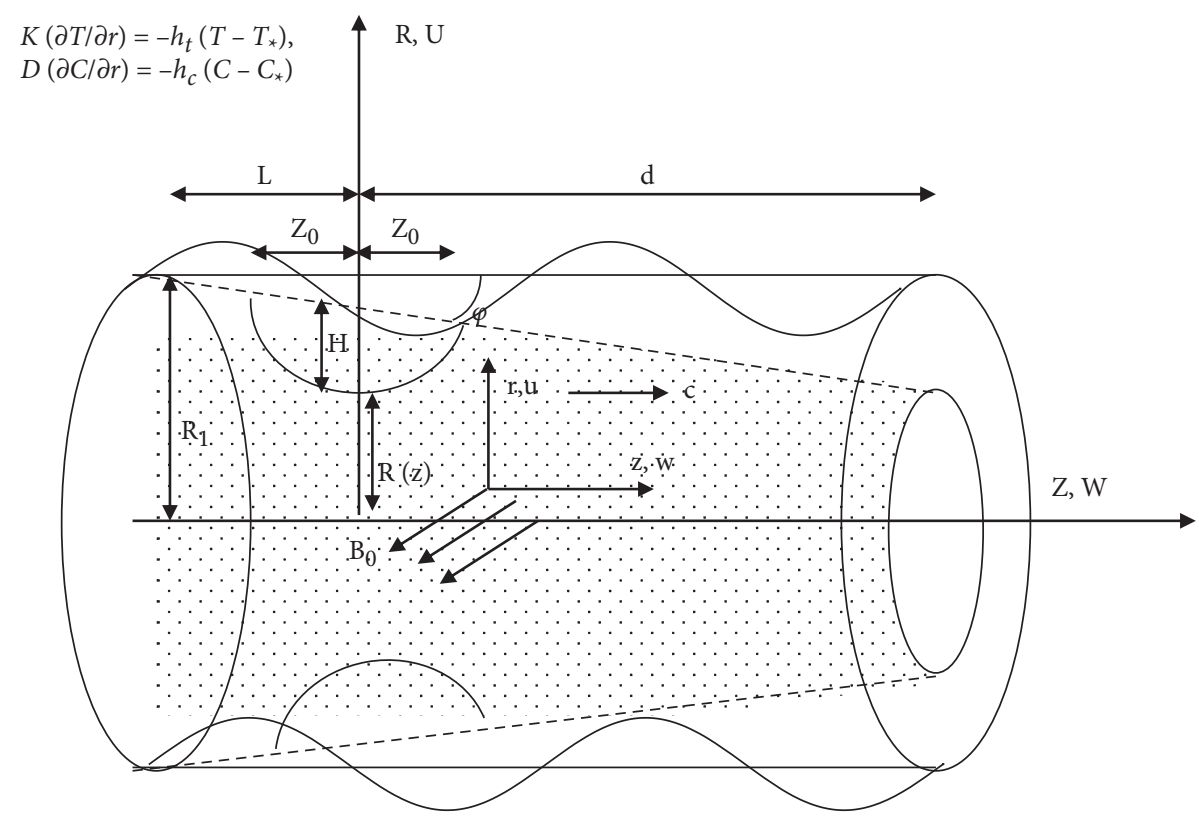

FIGURE 1: Sketch of the physical situation of the problem.

value of $a$. In accordance with the values $a$, there are three different cases of fluid as follows: the shear thickening fluids happen at $a<0$, Newtonian fluid occurs when $a=0$, and shear thinning fluids exist at $a>0$.

Recently, some fractal rheological models have appeared to describe non-Newtonian fluids [47-49], using the twoscale fractal calculus $[50,51]$.

In one-dimensional Rabinowitsch fluid [1], $\tau_{R R}, \tau_{\theta \theta}$, and $\tau_{Z Z}$ may be neglected. It follows that the constitutive equation (2) can be written as

$$
\mu\left(\frac{\partial W}{\partial r}+\frac{\partial U}{\partial z}\right)=\tau_{R Z}+a \tau_{R Z}^{3}
$$

For an incompressible fluid, the continuity equation yields

$$
\nabla . \underline{V}=0 \text {. }
$$

The governing equation of motion $[5,26]$ can be written as

$$
\rho\left(\frac{\partial \underline{V}}{\partial t}+(\underline{V} \cdot \nabla) \underline{V}\right)=-\nabla P+\nabla \cdot \underline{\tau}+\underline{J} \wedge \underline{B}-\frac{\mu}{k_{1}} \underline{V}-\frac{c_{f}}{\sqrt{k_{1}}} \underline{V}|\underline{V}|,
$$

where $P$ is the pressure, $J \wedge \underline{B}$ is Lorentz force, $J$ is the current density, $\underline{B}=\left(0, B_{0}, 0\right)$ is the external magnetic field, $k_{1}$ is the permeability of porous media, and $c_{f}$ is the Forchheimer coefficient. As shown by Krishna and Swarnalathamma [26], the current density can be introduced under the presumption of no polarization voltage (i.e., total electric field $\underline{E}=0$ ). Therefore, one finds

$$
\underline{J}=\sigma\left(\underline{V} \wedge \underline{B}-\frac{1}{e n_{c}} \underline{J} \wedge \underline{B}\right)
$$

where $\sigma$ is the electric conductivity, $e$ can be defined as the electron charge, $n_{c}$ represents the number density of free electrons, and $1 / e n_{c}$ is defined as the Hall factor. According to Arora [14] as well as Hayat et al. [19, 20], the equation of the energy thermal radiation and Dufour effects is represented as follows:

$$
\rho c_{p}\left(\frac{\partial T}{\partial t}+\underline{V} \cdot \nabla T\right)=\Phi_{1}+K \nabla^{2} T-\nabla \cdot q_{r}+\frac{1}{\sigma} \underline{J} \cdot \underline{J}+\frac{D K_{T}}{C_{s}} \nabla^{2} C+\frac{a_{1}}{k_{1}}(U+W)|\underline{V}|
$$

where $T$ is temperature, $k=K / \rho c_{p}$ is the thermometric conductivity, $K$ is the thermal conductivity, $c_{p}$ is the specific heat, $\Phi_{1}=S_{i j}\left(\partial V_{i} / \partial X_{j}\right)$ is the dissipation factor, $K_{T}$ is the thermal diffusion ratio, $C_{s}$ is the concentration susceptibility, $C$ is the concentration distribution, $|\underline{V}|=\sqrt{U^{2}+W^{2}}$, and $q_{r}$ is the radiative heat flux.

Employing the Rosseland diffusion flux model [52], the integrodifferential equation for the radiative transfer may be 
converted to a Fourier-type diffusion equation. It is to be noted that the Rosseland model is quite delicate in case of thermal radiation (scattering or absorption). The refractive index of the fluid-particle suspension is postulated to be uniform. Therefore, the nonlinear radiative heat flux may be imposed effectively as

$$
q_{r}=-\frac{4 \sigma_{0}}{3 K_{0}} \frac{\partial T^{4}}{\partial r}
$$

where $K_{0}$ is the Rosseland absorption coefficient and $\sigma_{0}$ is the Stefan-Boltzmann constant.

The temperature differences are supposed to be adequately small. Therefore, $T^{4}$ can be expressed as a linear function of temperature. By using Taylor series for $T^{4}$ about the mean fluid temperature $T_{m}$ and disregarding the higherorder terms, the nonlinear radiative heat flux is turned to the following linear form:

$$
q_{r}=-\frac{16 \sigma_{0}}{3 K_{0}} T_{m} \frac{\partial T}{\partial r}
$$

The equation of concentration with chemical reaction and Soret effects [19] may be represented as follows:

$$
\frac{\partial C}{\partial t}+\underline{V} \cdot \nabla C=D \nabla^{2} C+\frac{D K_{T}}{T_{m}} \nabla^{2} T-L_{c}\left(C-C^{*}\right),
$$

where $D$ is the coefficient of thermal diffusivity, $K_{T}$ is the thermal diffusion, $T_{m}$ is the mean fluid temperature, and $L_{c}$ is the reaction rate constant.

The geometry of the peristaltic wall surface may be defined as follows [7]:

$$
H_{1}=a_{0} \cos \frac{2 \pi}{\lambda}\left(Z-\frac{k t}{R_{1}}\right)
$$

where $a_{0}$ is the wave amplitude, $\lambda$ is the wave length, and $k t / R_{1}$ is the wave speed.

The convective boundary conditions with partial slip for the considered flow analysis $[20,30]$ can be listed as follows:

$$
\begin{aligned}
U & =\frac{\partial H_{1}}{\partial t}, \\
W & =-\gamma \tau_{R Z}, \\
K \frac{\partial T}{\partial r} & =-h_{t}\left(T-T_{*}\right), \\
D \frac{\partial C}{\partial r} & =-h_{c}\left(C-C_{*}\right), \text { at } R=R_{2}=R(z)+H_{1}, \\
U & =0, \\
\frac{\partial W}{\partial R} & =0, \\
\frac{\partial T}{\partial R} & =0, \\
\frac{\partial C}{\partial R} & =0, \text { at } R=0,
\end{aligned}
$$

where $\gamma$ is the slip parameter. $h_{t}$ is defined as the heat transfer coefficient, and it characterized the proportionality constant among heat flux and thermodynamic driving force for the heat flow. Meanwhile, $h_{c}$ describes the mass transfer coefficient, and it is explained to figure out the ratio between the actual mass flux of a species into or out of a flowing fluid and the driving force for this flux.

The instantaneous volume flow rate in the fixed coordinate system is determined as

$$
Q=2 \pi \int_{0}^{R_{2}} W R d R
$$

where $R_{2}$ is a function of $Z$ and $t$.

The time averaged $\widehat{Q}$ (time mean flow) over period $\tau=$ $\left(\lambda R_{1} / k\right)$ at a fixed $Z$-position is defined as

$$
\widehat{Q}=\frac{1}{\tau} \int_{0}^{\tau} Q \mathrm{~d} \tau
$$

It is assumed that the tube length is an integral multiple of wavelength $\lambda$. Additionally, the pressure difference through the ends of the tube is taken as a constant. So, the unsteady flow in the laboratory frame $(R, 0, Z)$ converts to a steady flow in the wave frame $(r, 0, z)$. The transformation between these two frames may be written as

$$
\begin{aligned}
& u=U, \\
& w=W-\frac{k}{R_{1}}, \\
& r=R, \\
& z=Z-\frac{k}{R_{1}} t .
\end{aligned}
$$

It is convenient to reformulate the previous equations in a proper nondimensional form. This can be done in a number of methods, depending primarily on the choice of the characteristics: length and mass. Consider the following nondimensional forms, counting on these characteristics as length $R_{1}, \lambda$ and mass $M$. The other nondimensional quantities are listed as follows:

$$
\begin{gathered}
\bar{r}=\frac{r}{R_{1}}, \\
\bar{z}=\frac{z}{\lambda}, \\
\bar{u}=\frac{u R_{1}}{k \delta}, \\
\bar{w}=\frac{w R_{1}}{k}, \\
\overline{H_{1}}=\frac{H_{1}}{R_{1}},
\end{gathered}
$$




$$
\begin{aligned}
\delta & =\frac{R_{1}}{\lambda}, \\
\bar{S} & =\frac{S R_{1}^{2}}{a_{1} k}, \\
\overline{z_{0}} & =\frac{z_{0}}{\lambda}, \\
\bar{L} & =\frac{L}{\lambda}, \\
\bar{h} & =\frac{h}{R_{1}}, \\
\bar{d} & =\frac{d}{\lambda}, \\
\overline{R(z)} & =\frac{R(z)}{R_{1}}, \\
\bar{P} & =\frac{P R_{1}^{3}}{a_{1} \lambda k}, \\
\bar{T} & =\frac{T-T^{*}}{\beta R_{1}}, \\
\bar{C} & =\frac{C-C^{*}}{C^{*}}, \\
\bar{Q} & =\frac{Q}{2 \pi R_{1} k} .
\end{aligned}
$$

Consider the other nondimensional parameters as follows: $R_{b}^{*}=\mu^{2} k^{2} a / R_{1}^{4}$ is the Rabinowitsch parameter, $D_{a}=k_{1} / R_{1}^{2}$ is the Darcy coefficient, $F_{r}=c_{f} R_{1} k / \nu \sqrt{k_{1}}$ is the Forchheimer number, $R_{e}=k / v$ is the Reynold number, $\nu=\mu / \rho$ is the kinematic viscosity, $\varepsilon=a_{0} / R_{1}$ is the amplitude ratio, $H^{2}=\sigma B_{0}^{2} R_{1}^{2} / \mu$ is the Hartmann number, $m=$ $\sigma B_{0} / e n_{c}$ is the Hall current parameter, $P_{r}=\mu / \rho k$ is the Prandtl number, $\beta$ is the adverse temperature gradient, $E_{c}=k^{2} / R_{1}^{3} c_{p} \beta$ is the Eckert number, $B_{r}=P_{r} E_{c}$ is the Brinkman number, $R_{n}=1+\left(16 \sigma_{0} T^{* 3} / 3 K K_{0}\right)$ is the radiation parameter, $D_{u}=D K_{T} C^{*} / \mu C_{s} \beta R_{1} c_{p}$ is the Dufour number, $S_{c}=k / D$ is the Schmidt number, $S_{r}=D K_{T} \beta R_{1} / k T_{m} C^{*}$ is the Soret number, $\alpha=L_{c} R_{1}^{2} / k$ is the chemical reaction number, $\gamma^{*}=\gamma \mu / R_{1}$ is the nondimensional slip parameters, $\beta_{t}=h_{t} R_{1} / K$ is the heat transfer Biot number, and $\beta_{c}=h_{c} R_{1} / D$ is the mass transfer Biot number. The bars mark indicates the nondimensional quantities. From now on, these will be canceled for simplicity.

According to the hypothesis of Verma and Parihar [12], we shall postulate that the radial velocity $u$ is very small in agreement with the axial one $w$. Further, the variation in the $z$-direction is more minimal than that in the radial one. Therefore, we may postulate that $u \ll w$ and $(\partial w / \partial z) \ll(\partial w / \partial r)$. Moreover, the assumption of long wavelength approximation $\delta \ll 1$ is also postulated. Now, $\delta$ is very little so that it can tend to zero. Consequently, the nondimensional governing equations (4), (6)-(8), and (11) with the appropriate boundary conditions (13) and (14) may be expressed as follows.

The equation of stress tensor is given by

$$
\frac{\partial w}{\partial r}=\tau_{r z}+R_{b} \tau_{r z}^{3} .
$$

The $r$-component of the velocity gives

$$
\frac{\partial P}{\partial r}=0 .
$$

Equation (20) indicates that $P$ is only a function of $z$. The $z$-component yields

$$
\frac{\mathrm{d} P}{\mathrm{~d} z}=\frac{1}{r} \frac{\partial}{\partial r}\left(r \tau_{r z}\right)-\left(\frac{H^{2}}{m^{2}+1}+\frac{1}{D_{a}}\right)(w+1)-F_{r}(w+1)^{2} .
$$

The heat equation results in

$$
\frac{1}{r} \frac{\partial}{\partial r}\left(r \frac{\partial T}{\partial r}\right)+\frac{D_{u} P_{r}}{1+R_{n}} \frac{1}{r} \frac{\partial}{\partial r}\left(r \frac{\partial C}{\partial r}\right)+\frac{B_{r}}{1+R_{n}}\left(\tau_{r z} \frac{\partial w}{\partial r}+\left(\frac{H^{2}}{m^{2}+1}+\frac{1}{D_{a}}\right)(w+1)^{2}\right)=0
$$

The concentration equation provides

$$
\frac{1}{r} \frac{\partial}{\partial r}\left(r \frac{\partial C}{\partial r}\right)+\frac{S_{r} S_{c}}{r} \frac{\partial}{\partial r}\left(r \frac{\partial T}{\partial r}\right)-\alpha S_{c} C=0 .
$$

The nondimensional volume flow rate in the moving coordinate system becomes

$$
q=\int_{0}^{r_{2}} w r d r .
$$


The nondimensional boundary conditions then become

$$
\begin{aligned}
u & =2 \pi \varepsilon \sin 2 \pi z, \\
w & =-1-\gamma^{*} \tau_{r z}, \\
\frac{\partial T}{\partial r} & =-\beta_{t} T, \\
\frac{\partial C}{\partial r} & =-\beta_{c} C, \text { at } r=r_{2}=r(z)+H_{1}, \\
u & =0, \\
\frac{\partial w}{\partial r} & =0, \\
\frac{\partial T}{\partial r} & =0, \\
\frac{\partial C}{\partial r} & =0, \text { at } r=0,
\end{aligned}
$$

where

$$
H_{1}=\varepsilon \cos 2 \pi z .
$$

The nondimensional effective radius of the tube $R(z)$ becomes

$$
R(z)= \begin{cases}1-m_{1}(z+L+d), & -L \leq z<-z_{0}, \\ 1-m_{1}(z+L+d)-\frac{h \cos \phi}{2}\left[1+\cos \frac{\pi z}{L_{0}}\right], & -z_{0} \leq z \leq z_{0}, \\ 1-m_{1}(z+L+d), & z_{0}<z \leq d .\end{cases}
$$

The next section is devoted to introducing the analytical solutions of these equations by means of HPM.

\section{Method of Solution}

The mixture of the HPM and the other classical perturbation methods showed that the former is more powerful, promising, and effective. This method excludes the obstacles that exist in the traditional classical perturbation methods.
Additionally, all advantages are kept. The HPM was first postulated by Ji-Huan He [53]. The HPM does not need any small parameter in the given equation. The HPM presumes an embedded parameter $p_{h} \in[0,1]$, which is taken to be small. Following [53-55], the HPM is adopted to the nonlinear differential equations (19)-(24) along with the boundary conditions as follows:

$$
\begin{aligned}
& H\left(\tau_{r z}, p_{h}\right)=L\left(\tau_{r z}\right)-L\left(\tau_{0 r z}\right)+p_{h} L\left(\tau_{0 r z}\right)+p_{h}\left[-\left(\frac{H^{2}}{m^{2}+1}+\frac{1}{D_{a}}\right)(w+1)-F_{r}(w 1)^{2}\right] \\
& H\left(T, p_{h}\right)=L(T)-L\left(T_{0 h}\right)+p_{h} L\left(T_{0 h}\right)+p_{h}\left[F_{r}+\frac{D_{u} P_{r}}{1+R_{n}} \frac{1}{r} \frac{\partial}{\partial r}\left(r \frac{\partial C}{\partial r}\right)+\frac{B_{r}}{1+R_{n}}\left(\tau_{r z} \frac{\partial w}{\partial r}+\left(\frac{H^{2}}{m^{2}+1}+\frac{1}{D_{a}}\right)(w+1)^{2}\right)\right] \\
& H\left(C, p_{h}\right)=L(C)-L\left(C_{0 h}\right)+p_{h} L\left(C_{0 h}\right)+p_{h}\left[-S_{r} S_{c}+\frac{S_{r} S_{c}}{r} \frac{\partial}{\partial r}\left(r \frac{\partial T}{\partial r}\right)-\alpha S_{c} C\right]
\end{aligned}
$$

To get the initial guesses for the stress $\tau_{0 r z}$, the linear operator $L$ may be taken as $L=(1 / r)(\partial / \partial r)$ $(r(\partial / \partial r))-(\mathrm{d} P / \mathrm{d} z)$. Hence, the initial guesses $\tau_{0 r z}$ can be defined as

$$
\tau_{0 r z}(r, z)=G(z) \frac{r}{2},
$$

where $G(z)=\mathrm{d} P / \mathrm{d} z$.

The guessing solution of velocity $w_{0 h}$ can be written as 


$$
w_{0 h}(r, z)=G(z)\left(\frac{r^{2}-r_{2}^{2}}{4}-\frac{\gamma^{*} r_{2}}{2}\right)+R_{b}[G(z)]^{3}\left(\frac{r^{4}-r_{2}^{4}}{32}\right)-1 .
$$

The solution $w_{0 h}(r, z)$ represents the velocity distribution in case of original flow (i.e., flow before the peristalsis occurs), which is presumed to be local Hagen-Poiseuille flow. Given that $\gamma^{*}=0, H^{2}=0, F_{r}=01 / D a \longrightarrow 0, h=0$, and $m_{1}=0$, the velocity distribution results in a good agreement with the previous work obtained by Singh and Singh [8] and Singh [9].

To get the initial guesses for the temperature distribution $T_{0 h}$, the linear operator $L$ may be considered as $L=(1 / r)(\partial / \partial r)(r(\partial / \partial r))-F_{r}$. Hence, the initial guesses $T_{0 h}$ can be defined as

$$
T_{0 h}(r, z)=F_{r}\left(\frac{r^{2}-r_{2}^{2}}{4}-\frac{1}{\beta_{t}} \frac{r_{2}}{2}\right)
$$

However, the linear operator $L$ may be taken as $L=$ $\left(L_{1} / r\right)(\partial / \partial r)(r(\partial / \partial r))+S_{c} S_{r}$ in order to determine the initial guesses for the concentration $C_{0 h}$. Consequently, the initial guesses $C_{0 h}$ can be expressed as

$$
C_{0 h}(r, z)=-S_{c} S_{r}\left(\frac{r^{2}-r_{2}^{2}}{4}-\frac{1}{\beta_{c}} \frac{r_{2}}{2}\right) \text {. }
$$

The basic presumption is that the solutions of equations (19) and (29)-(31) with the concerned boundary conditions (25) and (26) can be extended as a power series in $p_{h}$ :

$$
\begin{gathered}
w(r, z)=w_{0 h}+p_{h} w_{1 h}+\cdots, \\
\tau_{r z}(r, z)=\tau_{0 r z}+p_{h} \tau_{1 r z}+\cdots, \\
T(r, z)=T_{0 h}+p_{h} T_{1 h}+\cdots, \\
C(r, z)=C_{0 h}+p_{h} C_{1 h}+\cdots
\end{gathered}
$$

Applying the same technique as done by [53, 54], the solutions to shear stress, axial velocity, temperature, and concentration for $p_{h}=1$ can be, respectively, written as follows:

$$
\begin{aligned}
& \tau_{r z}(r, z)=\frac{r}{2} G(z)+G(z)\left(\frac{H^{2}}{m^{2}+1}+\frac{1}{D_{a}}\right)\left[\left(\frac{r^{3}}{16}+\frac{A_{5} r}{2}\right)+G^{2}(z) R_{b}\left(\frac{r^{5}}{192}-\frac{r_{2}^{2} r}{64}\right)\right] \\
& +F_{r} G^{2}(z)\left[\left(\frac{r^{5}}{96}+\frac{A_{1} r^{3}}{4}+\frac{A_{2} r}{2}\right)+G^{2}(z) R_{b}\left(\frac{r^{7}}{512}+\frac{A_{3} r^{4}}{5}-\frac{r_{2}^{2} r^{3}}{256}+\frac{A_{4} r}{2}\right)\right] \\
& +G^{6} F_{r}(z) R_{b}^{2}\left[\frac{r^{9}}{10240}-\frac{r_{2}^{4} r^{5}}{3072}+\frac{r_{2}^{8} r}{2048}\right] \\
& w(r, z)=G(z)\left(\frac{r^{2}-r_{2}^{2}}{4}-\frac{\gamma^{*} r_{2}}{2}\right)+R_{b}[G(z)]^{3}\left(\frac{r^{4}-r_{2}^{4}}{32}\right)-1+G(z)\left(\frac{H^{2}}{m^{2}+1}+\frac{1}{D_{a}}\right) \\
& \times\left[\left(\frac{r^{4}}{46}+\frac{A_{5} r^{2}}{4}\right)+G^{3}(z)\left(\frac{3}{4}\left(\frac{r^{6}}{96}+\frac{A_{5} r^{4}}{8}\right)+R_{b}\left(\left(\frac{r^{6}}{1152}-\frac{r_{2}^{4} r^{2}}{128}\right)+\frac{3}{4} G^{2}\left(\frac{r^{8}}{1536}-\frac{r_{2}^{4} r^{4}}{256}\right)\right)\right)\right] \\
& +F_{r} G^{2}(z)\left[\left(\frac{r^{6}}{621}+\frac{A_{1} r^{4}}{16}+\frac{A_{2} r^{2}}{4}\right)+\frac{3}{4} G^{2}(z)\left(\frac{r^{8}}{768}+\frac{A_{1} r^{6}}{24}+\frac{A_{2} r^{4}}{8}\right)\right] \\
& \times\left[\left(\frac{r^{4}}{46}+\frac{A_{5} r^{2}}{4}\right)+G^{3}(z)\left(\frac{3}{4}\left(\frac{r^{6}}{96}+\frac{A_{5} r^{4}}{8}\right)+R_{b}\left(\left(\frac{r^{6}}{1152}-\frac{r_{2}^{4} r^{2}}{128}\right)+\frac{3}{4} G^{2}\left(\frac{r^{8}}{1536}-\frac{r_{2}^{4} r^{4}}{256}\right)\right)\right)\right] \\
& +F_{r} G^{2}(z)\left[\left(\frac{r^{6}}{621}+\frac{A_{1} r^{4}}{16}+\frac{A_{2} r^{2}}{4}\right)+\frac{3}{4} G^{2}(z)\left(\frac{r^{8}}{768}+\frac{A_{1} r^{6}}{24}+\frac{A_{2} r^{4}}{8}\right)\right] \\
& +G^{4}(z) R_{b} F_{r}\left[\left(\frac{r^{8}}{4096}+\frac{A_{3} r^{5}}{25}-\frac{r_{2}^{2} r^{4}}{1024}+\frac{A_{4} r^{2}}{4}\right)+\frac{3}{4} G^{4}(z)\left(\frac{r^{10}}{5120}+\frac{A_{3} r^{7}}{35}-\frac{r_{2}^{2} r^{6}}{1536}+\frac{A_{4} r^{2}}{8}\right)\right] \\
& +G^{6}(z) F_{r} R_{b}^{2}\left[\left(\frac{r^{10}}{102400}-\frac{r_{2}^{4} r^{6}}{18432}+\frac{r_{2}^{8} r^{2}}{4096}\right)+\frac{3}{4} G^{4}(z) \times\left(\frac{r^{12}}{122880}-\frac{r_{2}^{4} r^{8}}{24576}+\frac{r_{2}^{8} r^{4}}{8192}\right)\right]+A_{6} \text {, } \\
& T(r, z)=\frac{D_{u} P_{r}}{1+R_{n}} \frac{r^{2}}{4}-\frac{B_{r} G^{2}(z)}{1+R_{n}}\left\{\left(\frac{r^{4}}{64}+R_{b} \frac{r^{6}}{576}\right)+\left(\frac{H^{2}}{m^{2}+1}+\frac{1}{D_{a}}\right)\left[\left(\frac{r^{6}}{576}+\frac{A_{1} r^{4}}{16}+\frac{A_{2} r^{2}}{4}\right)\right.\right. \\
& \left.\left.+G^{2}(z) R_{b}\left(\frac{r^{8}}{4096}+\frac{A_{3} r^{6}}{36}-\frac{r_{2}^{2} r^{4}}{1024}+\frac{A_{4} r^{2}}{4}\right)+G^{4}(z) R_{b}^{2}\left(\frac{r^{10}}{102400}+\frac{r_{2}^{4} r^{4}}{18432}+\frac{r_{2}^{8} r^{2}}{4096}\right)\right]\right\}+A_{15} \text {, }
\end{aligned}
$$




$$
C(r, z)=-S_{c} S_{r} F_{r} \frac{r^{2}}{4}+\alpha S_{c}\left(-S_{c} S_{r} \frac{r^{4}}{64}+\frac{A_{11} r^{2}}{4}\right)+A_{17}
$$

where the constants $A_{1}, A_{2}, \ldots, A_{17}$ are mentioned in the appendix.

Now, the volume flow rate is employed to get the pressure gradient $G(z)$.

By utilizing equations (15) and (24), one gets

$$
Q=q+\frac{r_{2}^{2}}{2}
$$

From equation (16),

$$
\widehat{Q}=q+\frac{1}{4}\left([2 R(z)]^{2}+\varepsilon^{2}\right) .
$$

With the help of equations (33), (44), and (45), the equation of pressure gradient can be expressed as

$$
R_{b} G^{3}(z)+\frac{A_{7}}{A_{8}} G(z)+\left(\frac{A_{9}-q}{A_{8}}\right)=\frac{1}{A_{7}} .
$$

For small values of $R_{b}$, equation (46) can be perturbed as follows:

$$
G(z)=G_{0}(z)+R_{b} G_{1}(z)+\cdots
$$

so that

$$
\begin{array}{r}
G(z)=\frac{\mathrm{d} P}{\mathrm{~d} z} \\
=\frac{1}{A_{7}}\left(\widehat{Q}-\frac{1}{4}\left(2[R(z)]^{2}-\varepsilon^{2}\right)+A_{9}\right)-\frac{A_{8} R_{b}}{A_{9} A_{7}^{3}}\left(\widehat{Q}-\frac{1}{4}\left(2[R(z)]^{2}-\varepsilon^{2}\right)+A_{9}\right)^{3} .
\end{array}
$$

For $\gamma^{*}=0, H^{2}=0, F_{r}=0,1 / D a \longrightarrow 0, h=0$, and $m_{1}=0$, the solution of pressure gradient (48) is reduced to

$$
G(z)=-8\left[\frac{2\left(\widehat{Q}-(1 / 4)\left(2(R(z))^{2}-\varepsilon^{2}\right)\right)+r_{2}^{2}}{r_{2}^{4}}\right]+R_{b} \frac{\left.256\left(2\left(\widehat{Q}-(1 / 4)\left(2[R(z)]^{2}-\varepsilon^{2}\right)\right)+r_{2}^{2}\right)\right)^{3}}{3 r_{2}^{10}}
$$

Equation (49) can be expressed in another form as follows:

$$
G(z)=\frac{\mathrm{d} P}{\mathrm{~d} z}=-8\left(\frac{2 q+r_{2}^{2}}{r_{2}^{4}}\right)+R_{b} \frac{256\left(2 q+r_{2}^{2}\right)^{3}}{3 r_{2}^{10}}
$$

which is in a good agreement with the previous work obtained by Singh and Singh [8].

The pressure rise $\Delta P$ and the friction force of the tube $\Delta F$, in their nondimensional forms, are given by

$$
\begin{aligned}
\Delta P= & \int_{-L}^{d}(G z) \mathrm{d} z \\
= & \int_{-L}^{-z_{0}}(G z) \mathrm{d} z+\int_{-z_{0}}^{z_{0}}(G z) \mathrm{d} z+\int_{z_{0}}^{d}(G z) \mathrm{d} z, \\
\Delta F= & \int_{-L}^{d} r_{2}^{2}(-G(z)) \mathrm{d} z \\
= & \int_{-L}^{z_{0}} r_{2}^{2}(-G(z)) \mathrm{d} z+\int_{-z_{0}}^{z_{0}} r_{2}^{2}(-G(z)) \mathrm{d} z \\
& +\int_{z_{0}}^{d} r_{2}^{2}(-G(z)) \mathrm{d} z .
\end{aligned}
$$

Because of the intricacy in evaluating these integrations, their values are numerically estimated.

In case of ignoring these parameters $\left(\gamma^{*}, H^{2}, F_{r}, D a, h\right.$, $m_{1}$, and $R_{b}$ ), the pressure rise and friction force result in good agreement with the previous work obtained by Shapiro et al. [5].

\section{Results}

4.1. Pumping Characteristics. A mathematical software is employed to indicate the quantitative impacts of different physical parameters that are involved in distributions of the axial velocity $w$, temperature $T$, concentration $C$, pressure rise $\Delta P$, and the friction force $\Delta F$. The deemed ranges of governing parameters are taken from previous works relevant to this research; see $[7,9,12]$. This range is prepared for various values of the parameters: $H^{2}=10, F_{r}=10$, $D_{a}=0.02, R_{b}=0.2, L=1, z_{0}=0.5, d=1, m=0.5, \gamma^{*}=0.8$, $\varepsilon=0.01, \quad \phi=0.05, h=0.1, \quad R_{n}=0.05, Q=0.1, S_{c}=0.3$, $S_{r}=0.5, D_{u}=0.1, P_{r}=2, \alpha=0.1, B_{r}=0.3, \beta_{t}=0.6$, and $\beta_{c}=0.4$. Some essential results are graphically exhibited in Figures 2-8. 


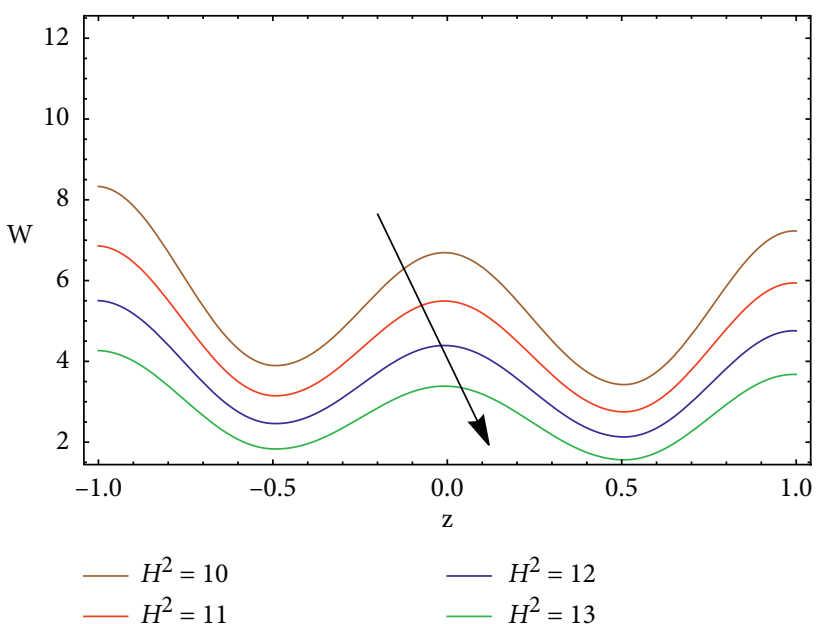

(a)

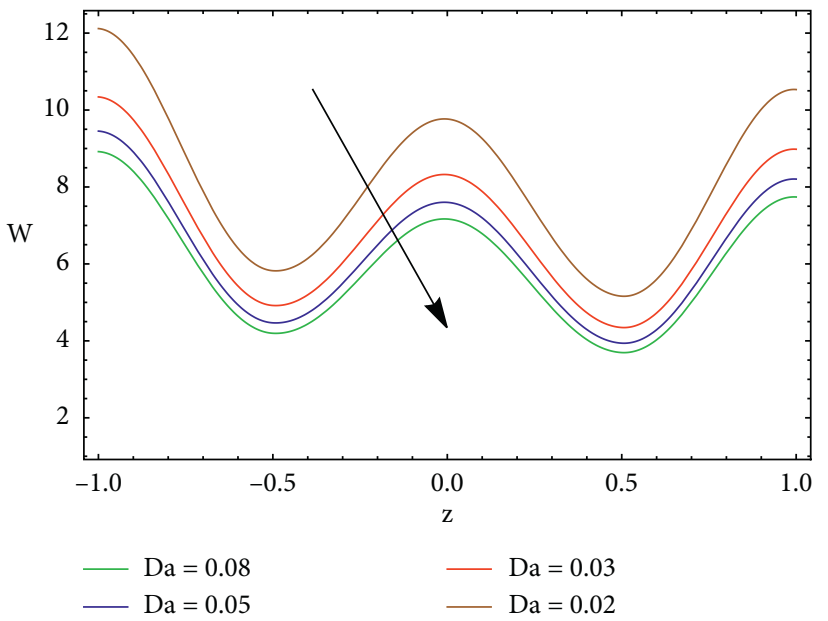

(c)

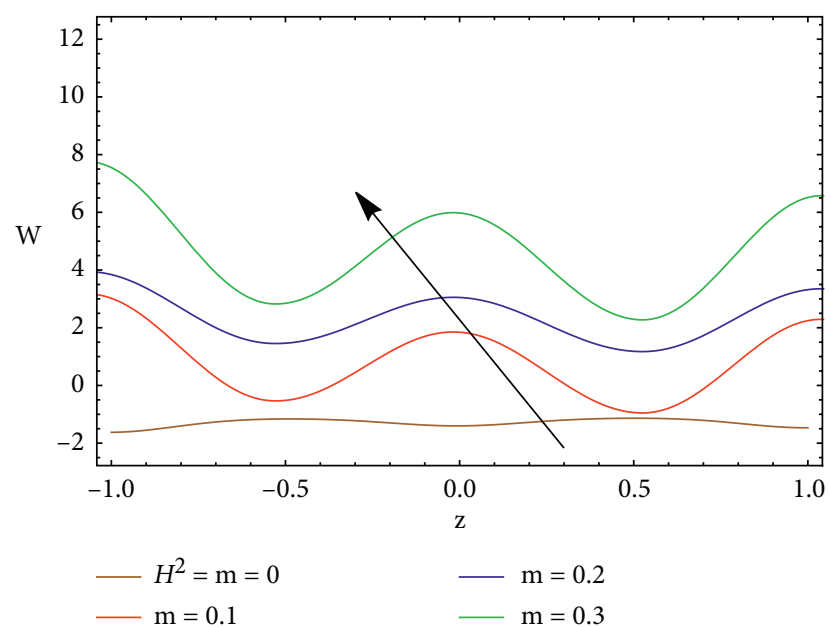

(b)

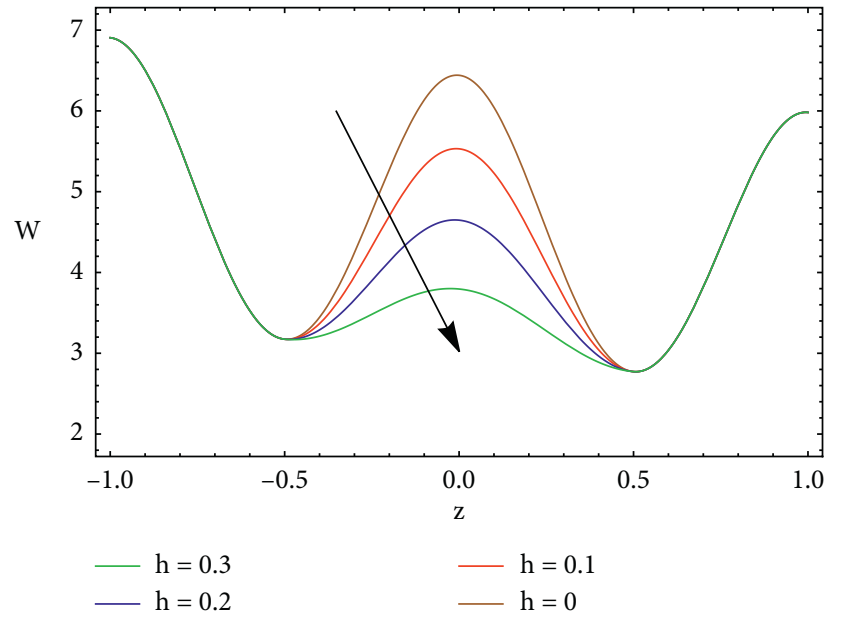

(d)

Figure 2: The variation of the axial velocity $w(z)$ for different values of $H^{2}, m, D_{a}$, and $h$.

Figure 2(a) describes the variation of axial velocity $w(z)$ for different values of the Hartmann number $H^{2}$. It is detected that the axial velocity diminishes with the enhancement of $H^{2}$. This phenomenon comes in light of the enhancement in the Hartmann number, giving rise to the Lorentz force. It is renowned that the Lorentz force contradicts the flow. This signalizes that if the strength of the magnetic field enhances, the flow of blood will be hindered.

Figure 2(b) displays the variation of axial velocity $w(z)$ for different values of Hall parameter $m$. This figure illustrates the increasing effect of axial velocity due to the enhancement of Hall parameter $m$. Actually, at larger values of $m$, the effective conductivity diminishes. It leads to a reduction in the magnetic damping force; consequently, the axial velocity is enhanced. Furthermore, it is signalized that the velocity is lower in case of disregarding the impacts of Hall current and Joule heating $\left(H^{2}=m=0\right)$.

Figure 2(c) illustrates the variation of axial velocity $w(z)$ for different values of Darcy coefficient $D_{a}$. There is a progressive reduction in the axial flow of velocity with enlarging $D_{a}$. The higher porosity parameter is connected to smaller permeability. Therefore, a small gap is obtainable for fluid to flow, which leads to a reduction in velocity. So, a porous medium behaves like a resistive force, which hinders the fluid velocity.

Figure 2(d) displays the variation of axial velocity $w(z)$ for different values of the maximum height of stenosis $h$. It is to be noted that the axial velocity diminishes with enlarging of $h$ at the domain $(-0.5 \leq z \leq 0.5)$. In the meantime, at the complementary domain, the curves of the velocity are applicable to each other. Additionally, in the case of no stenosis $(h=0)$, the values of the axial velocity are larger than those in case of stenosis. Therefore, for the diseases of blood clot, the presence of the clots at the artery hinders the blood flow, which has a harmful impact on the body organs [11].

Figure 3(a) indicates the variation of axial velocity $w(r)$ for different values of the Forchheimer number $F_{r}$. It is revealed that when the values of the Darcy-Forchheimer number $F_{r}$ enlarge, the velocity of the flow becomes weaker. In the state of a porous gap with larger pore sizes, enlarging $F_{r}$ enhances the viscous interference. Hence, the increase in $F_{r}$ leads to a better flow resistance, so the velocity of fluid 


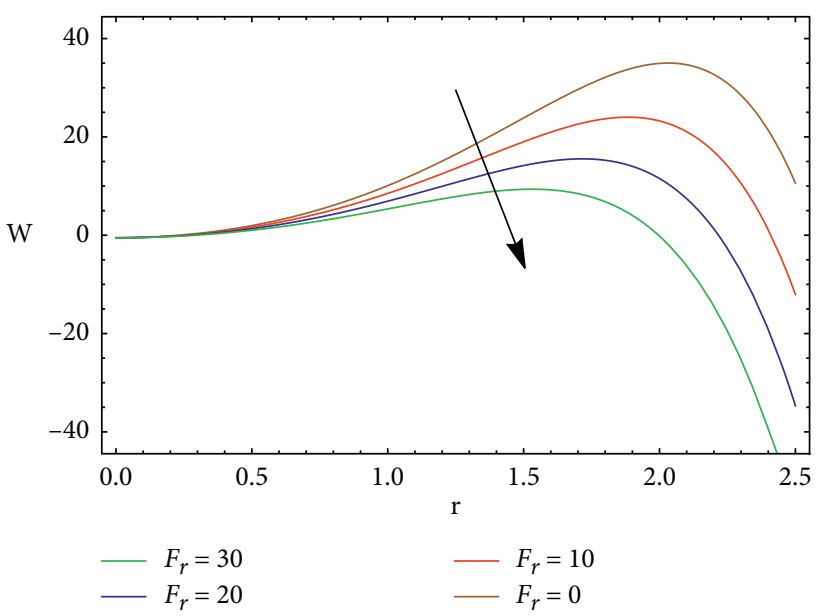

(a)

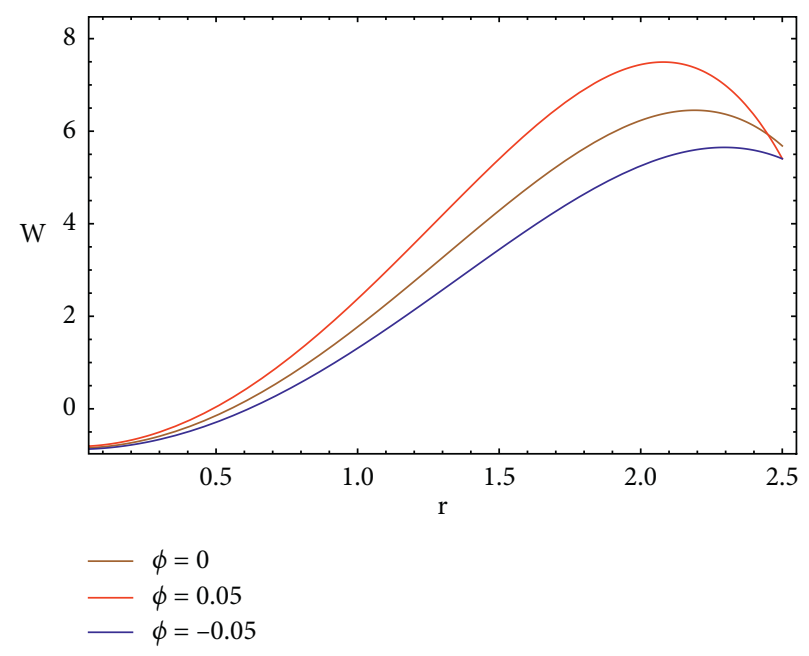

(c)

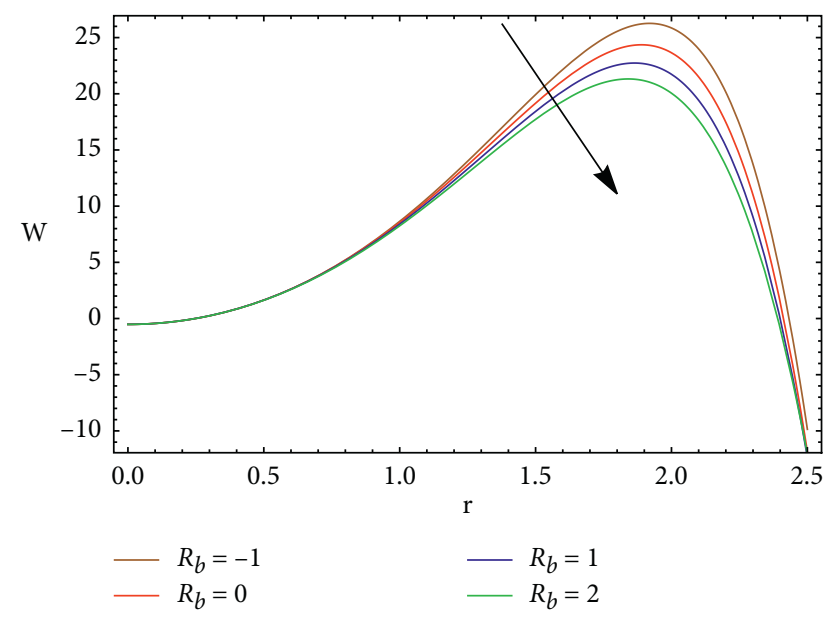

(b)

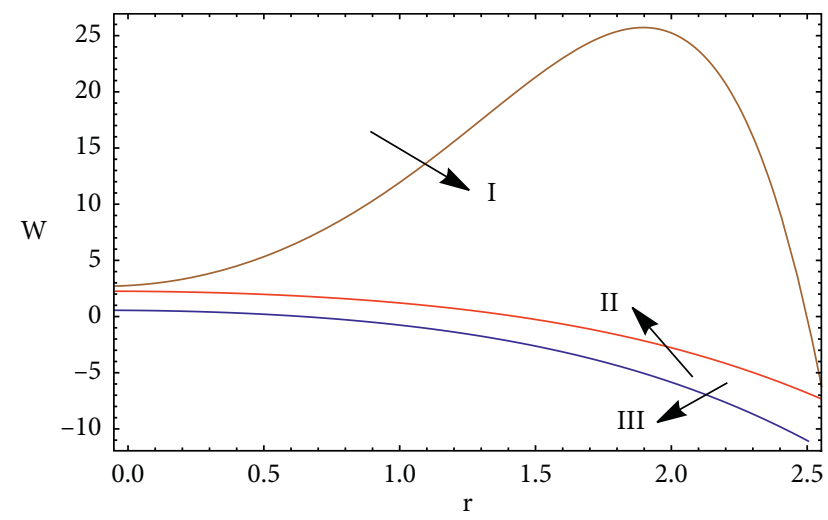

(d)

FIgURE 3: The variation of the axial velocity $w(r)$ for different values of $F_{r}, R_{b}, \varphi$ and special cases.

hinders. Therefore, the minimum velocity concerned with the maximum value of $F_{r}$, which elucidates that the Forchheimer quadratic drag strongly decelerates the flow rate. The results in case of the Darcy flow can be retrieved by setting $F_{r}=0$. It is observed that the values of axial velocity, in case of the Darcy flow, are lower than those in case of the Darcy-Forchheimer flow.

Figure 3(b) elucidates the variation of axial velocity $w(r)$ for different values of the Rabinowitsch parameter $R_{b}$. The effect of non-Newtonian fluids is analyzed by taking different fluids counting on the values of $R_{b}$. It is revealed that the axial velocity hinders with the increase of $R_{b}$ because the rise in the Rabinowitsch parameter $R_{b}$ results rise the elasticity in the fluid. This makes the fluid thicker and impedes the velocity of the fluid. Moreover, in case of dilatant fluids $\left(R_{b}<0\right)$, the values of the axial velocity are higher than those in both cases of the Newtonian fluid $\left(R_{b}=0\right)$ and the pseudoplastic fluids $\left(R_{b}>0\right)$.

Figure 3(c) reveals the variation of axial velocity $w$ versus the radial distance $r$ for different values of the taper angle $\phi$. The prominence of the impact of vessel tapering on the shape of stenosis deserves particular attention. Furthermore, the tapering has a considerable portion of the arterial system [12]. Consequently, the current work focuses on examining the flow along the tapered tube with stenosis. It is elucidated that in case of the diverging tapered artery $\phi=0.05(>0)$, the values of the axial velocity are higher than those in case of the nontapered artery $\phi=0$ and convergent tapered one $\phi=-0.05(<0)$.

Figure 3(d) shows a comparison between the current work and the previous works of Singh and Singh [8], which is plotted by the dashed curve $\left(F_{r}=0, \gamma^{*}=0, \phi=0, h=0\right.$, $B_{0}=0, R_{b}=0, R_{n}=0, m=0, H^{2}=0, T=0$, and $\left.C=0\right)$, and Singh [9] in the presence of temperature, which is represented in the figure by a dotted curve. It is observed that the values of axial velocity in our study are higher than those in the other two cases.

Figure 4(a) illustrates the variation of temperature $T(r)$ for different values of the Dufour number $D_{u}$ and the Soret number $S_{r}$. It is recognized that the temperature rises with the enhancement of both $D_{u}$ and $S_{r}$. In fact, the enlargement in both $D_{u}$ and $S_{r}$ results in a supplement in the thermal 


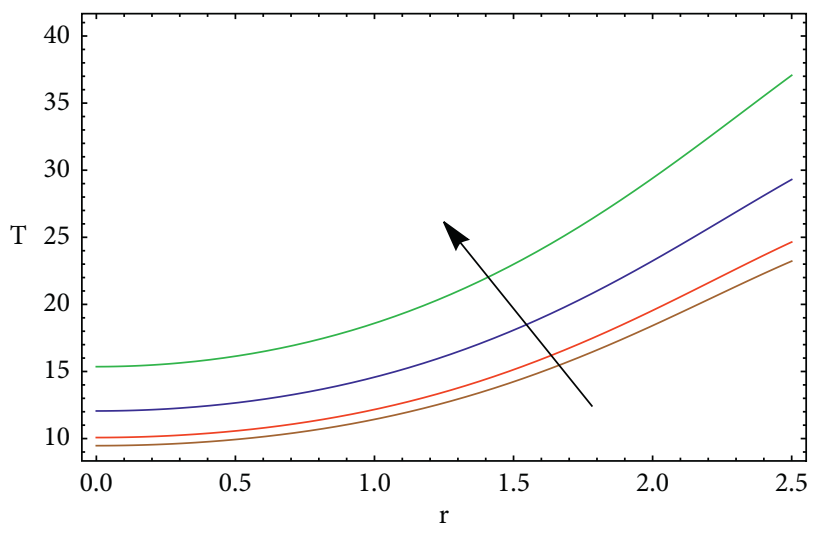

$$
\begin{aligned}
-S_{r} & =0.5, \mathrm{Du}=1 \\
-S_{r} & =2.0, \mathrm{Du}=3
\end{aligned}
$$

(a)

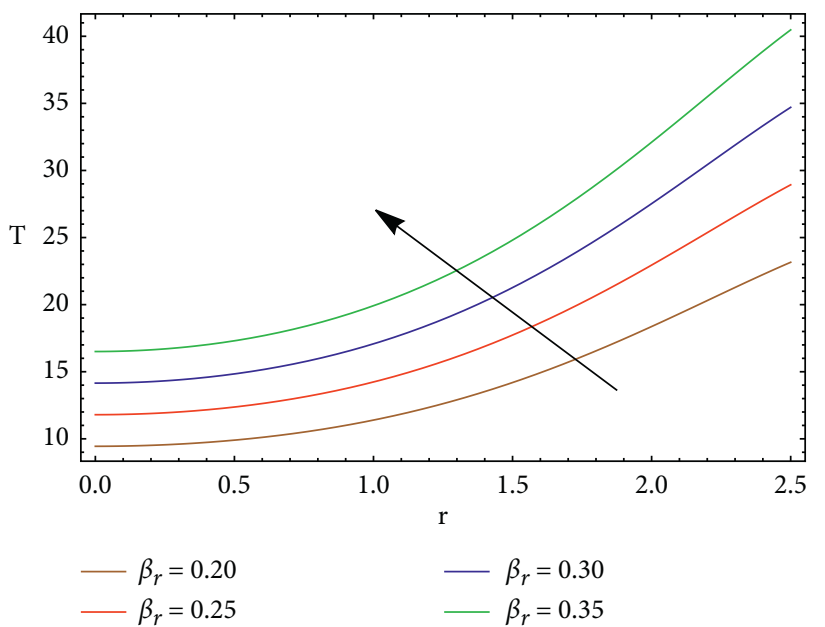

(c)

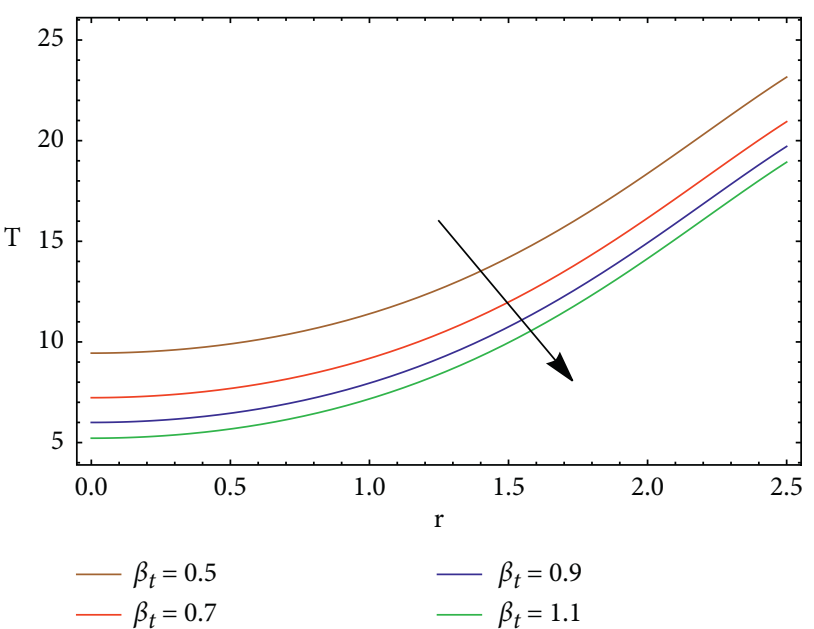

(b)

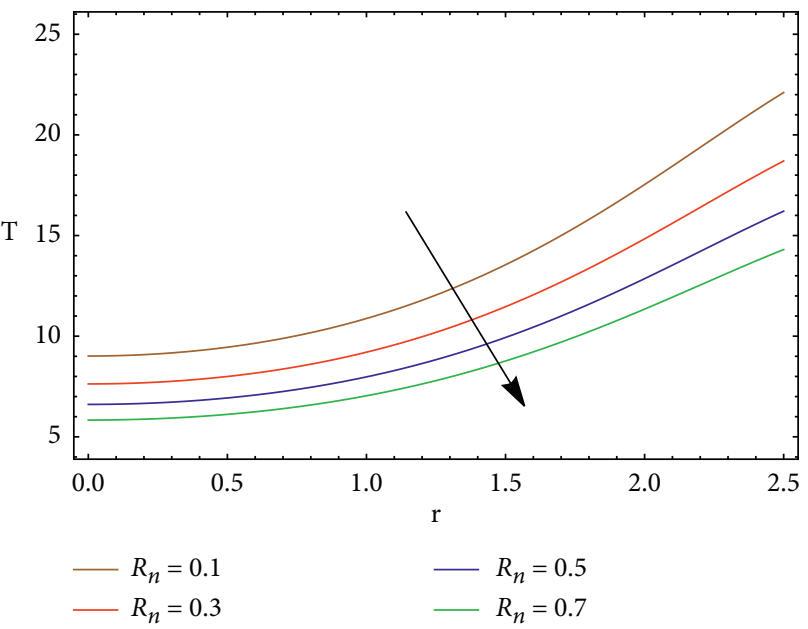

(d)

FIgURE 4: The variation of the temperature distribution $T(r)$ for different values of $D_{u}, S_{r}, \beta_{t}, B_{r}$, and $R_{n}$.

diffusion, and then the temperature rises. Physically, the diffusion-thermo or the Dufour influence is recognized as a heat flux conducted when a chemical system undergoes a concentration gradient. These influences are counted on thermal diffusion which is very small, but which sometimes becomes major due to the different molecular weights. Mass diffusion is followed by the disparate distribution of species producing a concentration gradient. Furthermore, a temperature gradient can be imposed as a driving force for mass diffusion, which is defined as thermodiffusion or Soret effect. Consequently, the enhancing Soret impact increases the temperature gradient. These results are in agreement with the earlier work of Hayat et al. [56].

Through Figure 4(b), the effect of heat transfer Biot number $\beta_{t}$ on temperature is described. It is revealed that the temperature is decelerated with the increase of $\beta_{t}$. The obtained result reveals that the thermal conductivity of the fluid reduces with the rise in $\beta_{t}$ and that reduces the temperature.

The variation of temperature profile $T(r)$ for different values of the Brinkman number $B_{r}$ is outlined in Figure 4(c).
The Brinkman number is recognized as a nondimensional number concerned with heat conduction from a wall to a flowing viscous fluid, which is employed in a polymer processing. It is also described as the ratio between heat produced by viscous dissipation and heat transported by molecular conduction. In a few words, it can introduced as the ratio of the viscous heat generation of external heating. It is observed that the nondimensional temperature is enhanced with the enlargement of $B_{r}$ value. This situation can be interpreted by higher thermal energy, which is produced by viscous dissipation. Therefore, the fluid temperature is enhanced. However, this behaviour makes some unexpected problems such as shrinkage, stretching, and deterioration of the arteries.

Figure 4(d) describes the variation of temperature $T$ versus the $r$-axis for different values of radiation parameter $R_{n}$. It is caused by the fact that the thermal radiation is inversely proportional with the thermal conduction. This reveals that maximum heat is radiated away from the system, which makes a reduction in the heat conduction of the fluid. Thermal radiation may be beneficial in metal coating. 


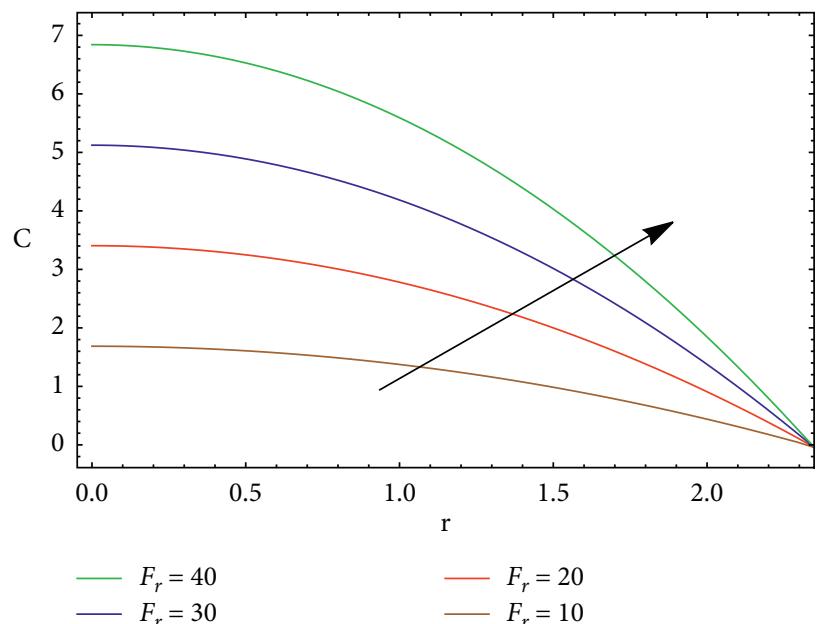

(a)

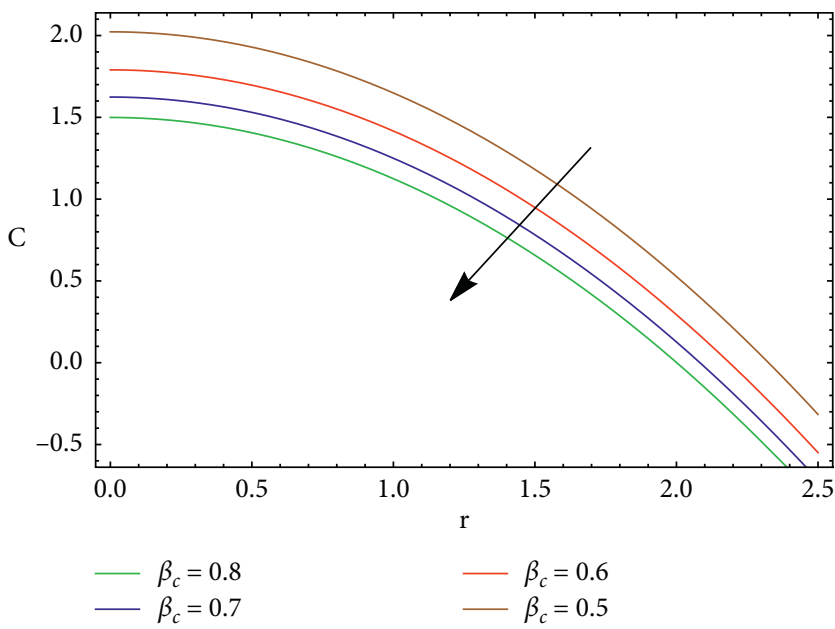

(c)

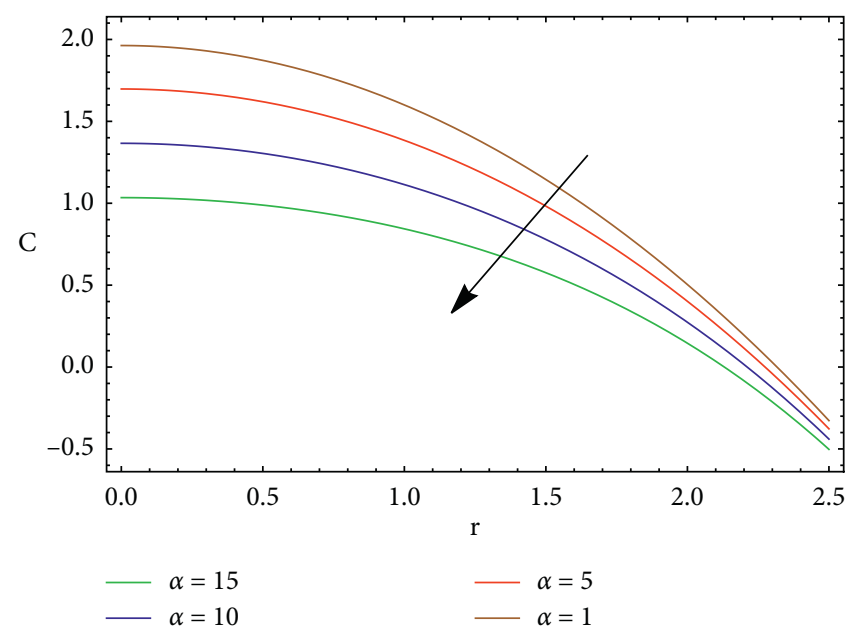

(b)

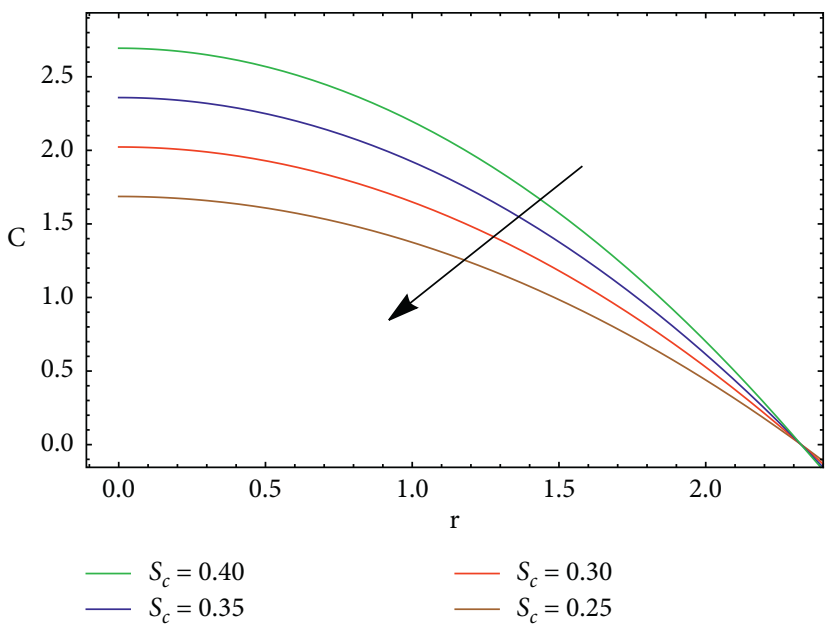

(d)

FIGURE 5: The variation of the concentration $C(r)$ for different values of $F_{r}, \alpha, \beta_{c}$, and $S_{c}$.

The variation of the concentration profile $C(r)$ for different values of the Darcy-Forchheimer number $F_{r}$ is displayed in Figure 5(a). It reveals that a rise in $F_{r}$ strengthens the nondimensional concentration. The decrease in velocity $u$ with the enhanced Forchheimer numbers $F_{r}$ implies that shear stresses increase because the shear stresses are proportional to the rising velocity gradient. Therefore, the flow experiences more retardation and this enlarges the shear stress serves as an insulation to the mass flux. Consequently, concentrations are enhanced.

Figure 5(b) describes the variation of concentration $C$ versus the $r$-axis for different values of chemical reaction number $\alpha$. Chemical reaction promotes the interfacial mass transfer rate, which causes a decrease in the concentration distribution.

Figure 5(c) reveals the variation of concentration $C(r)$ for different values of the mass transfer Biot number $\beta_{c}$. It is illustrated that the concentration impedes with the increase of $\beta_{c}$. The obtained result indicates that the mass conductivity of the fluid is weakened by the boost of $\beta_{c}$ and that leads to a reduction in concentration.
Figure 5(d) shows the variation of concentration $C$ versus $r$-axis for different values of the Schmidt number $S_{c}$. It is observed that the concentration profile diminishes when $S_{c}$ rises. The Schmidt number is recognized as the ratio of momentum diffusivity (viscosity) to mass diffusivity. Therefore, increasing $S_{c}$ causes reduction in the mass diffusion, which boosts the intermolecular force and consequently results in a reduction in concentration.

From all the pervious results, this study is very significant in many medical applications, such as the gastric juice motion in the small intestine and the flow of blood in arteries. The results show the effects of heat and mass transfer and some other parameters on the peristaltic motion, which have a mild stenosis. This mild stenosis can be considered as blood clots. This results can present the aid in medicine to solve some medical problems, such as breaking up blood clots.

Figures 6(a) and 6(b) describe the variation of the pressure gradient $\mathrm{d} P / \mathrm{d} z$ with $z$-axis for different values of the amplitude ratio $\varepsilon$ and the Rabinowitsch parameter $R_{b}$. It is depicted from these figures that for $z \in[-1,-0.7]$, 


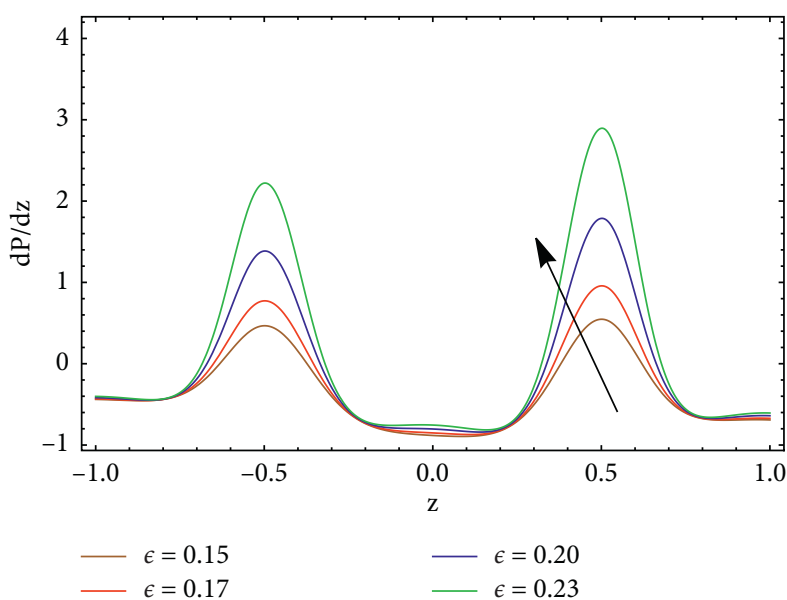

(a)

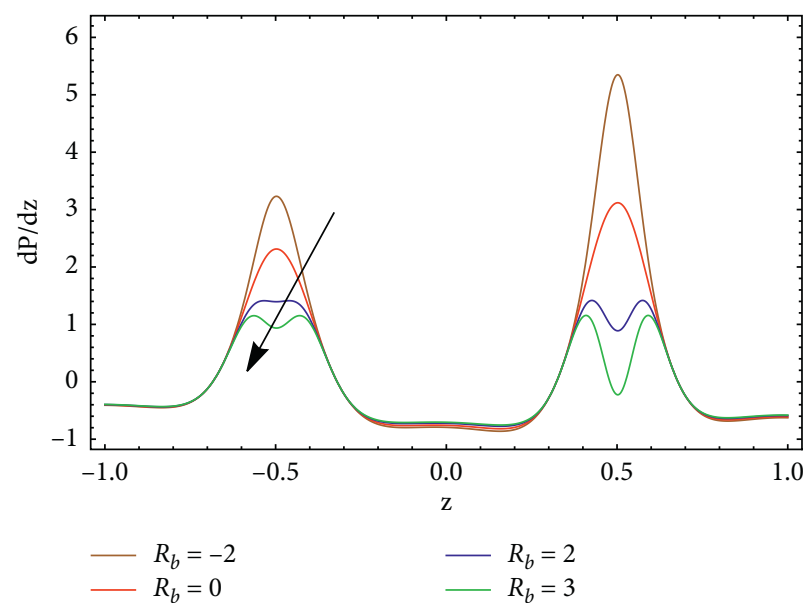

(b)

Figure 6: The variation of pressure gradient $\mathrm{d} P / \mathrm{d} z$ for different values of $\varepsilon$ and $R_{b}$.

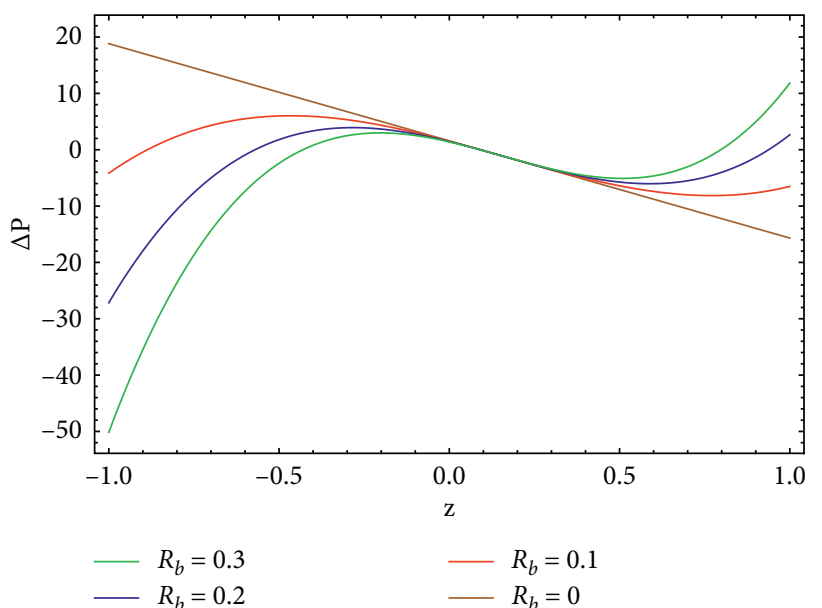

(a)

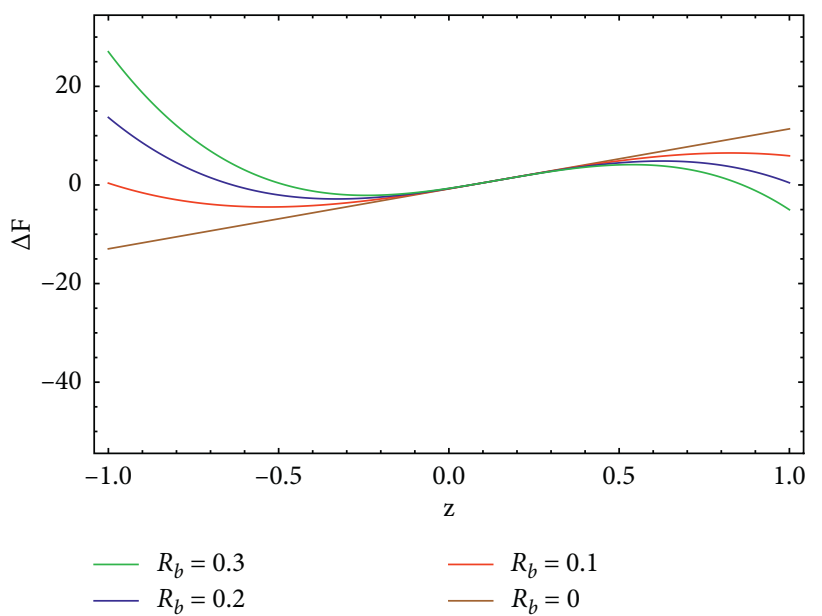

(c)

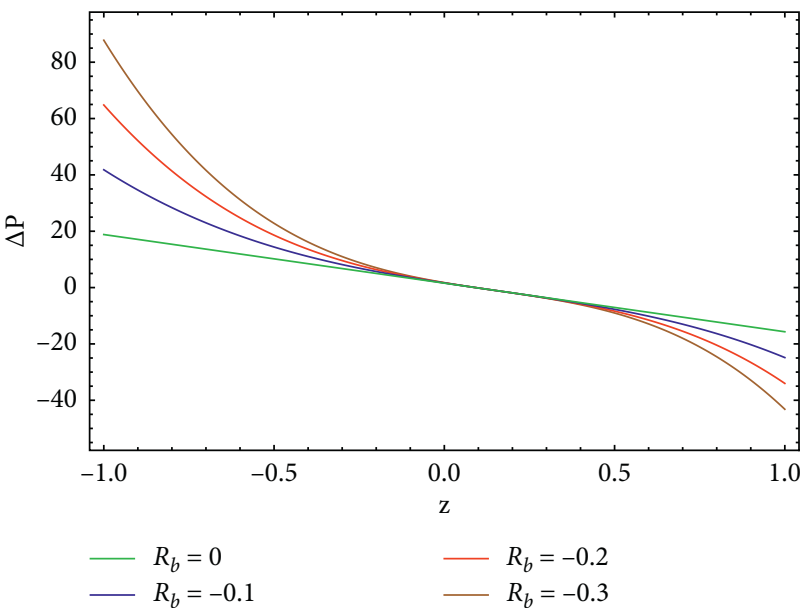

(b)

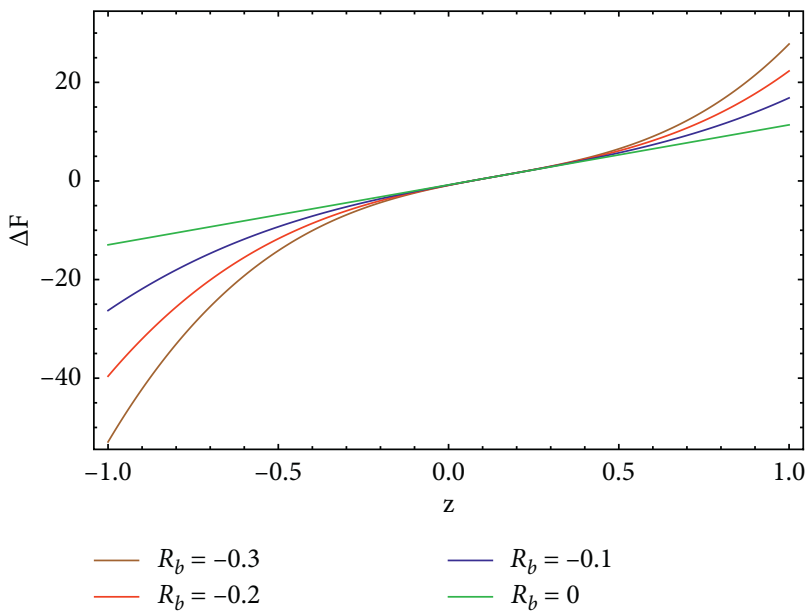

(d)

FIgURE 7: The variation of pressure rise $\Delta P$ and friction force $\Delta F$ versus mean flow rate for different values of $R_{b}$. 

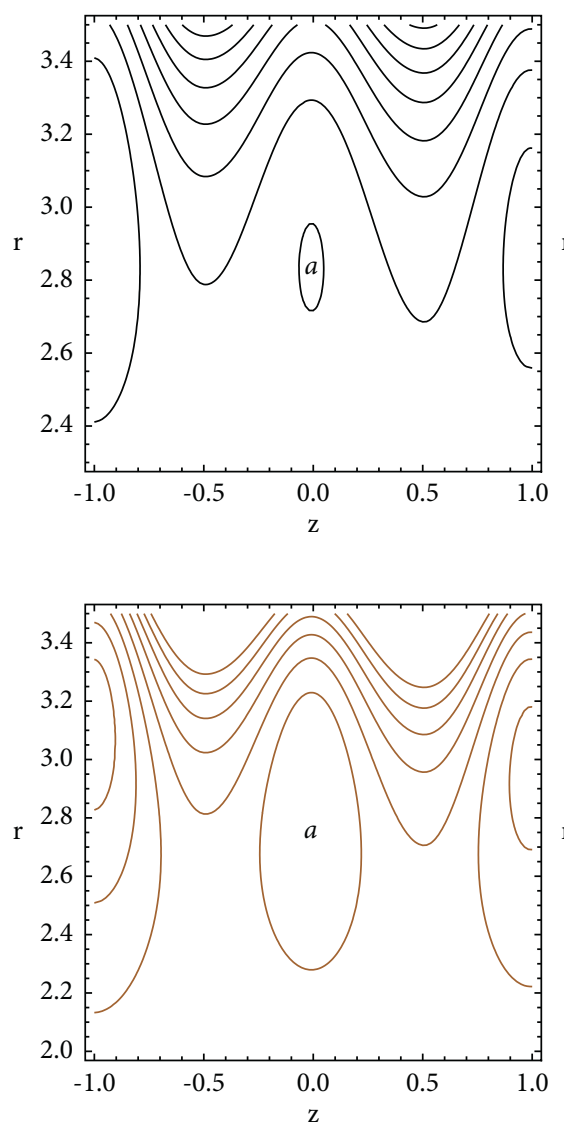
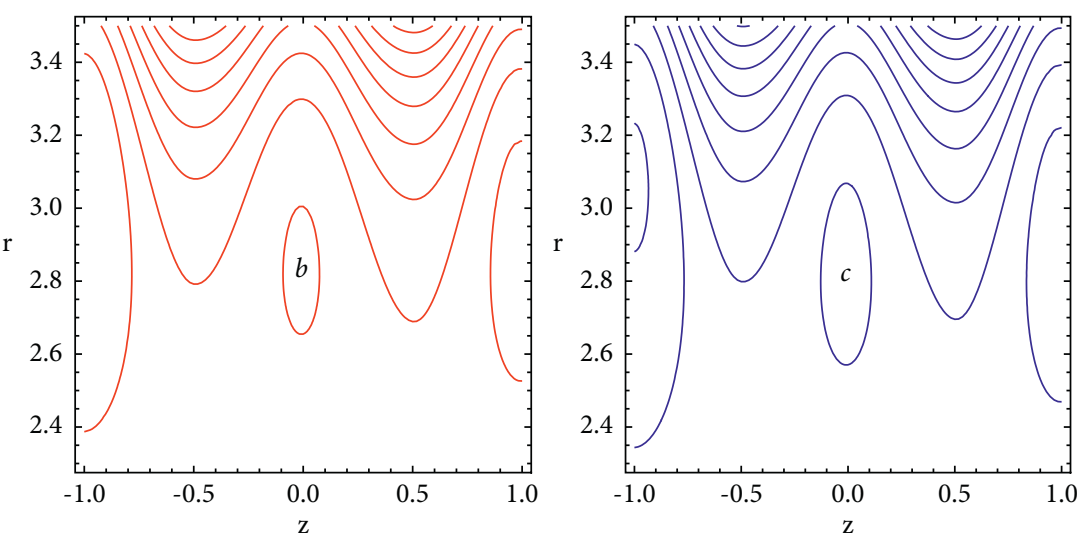

(a)
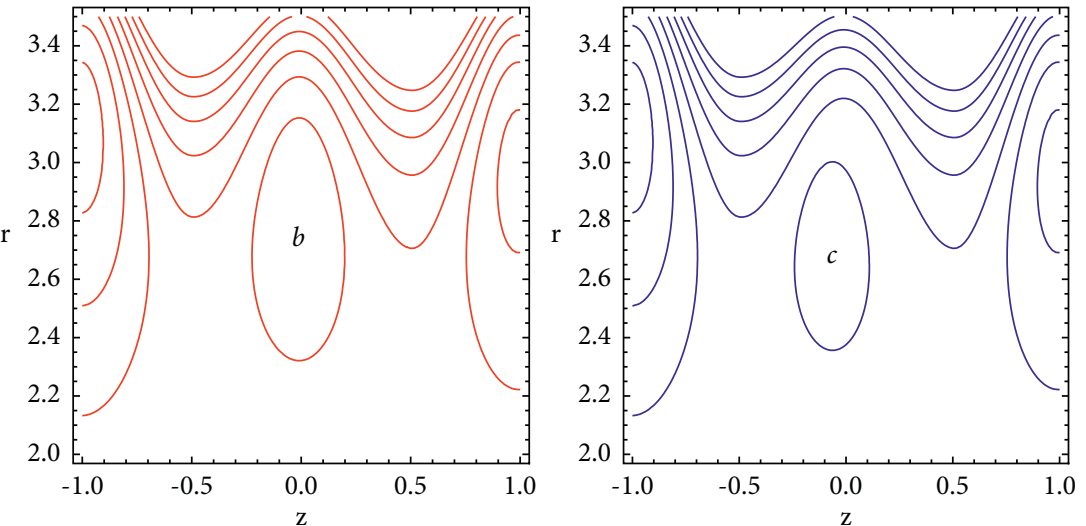

(b)

FIgURE 8: The streamlines' contour plot for different values of $h$ and $m$.

$z \in[-0.3,0.3]$, and $z \in[0.7,1]$, the pressure gradient becomes small; that is, the flow can easily stream without the imposition of a large pressure gradient. Meanwhile, in the regions $z \in]-0.7,-0.3$ [ and $z \in] 0.3,0.7$ [, the pressure gradient is enhanced with the enlargement of $\varepsilon$. The volume flow rate is proportional to the amplitude ratio, which produces an increase in the pressure gradient. However, the pressure gradient diminishes with the enhancement of $R_{b}$. The rise in the Rabinowitsch parameter $R_{b}$ raises elasticity of the fluid. Therefore, the pressure gradient is diminished. Furthermore, it is revealed that the non-Newtonian impact of cubic stress flow can generate different results to the pressure gradient. The comparison with the case of Newtonian fluids $R_{b}=0$ generally illustrates that the dilatant influences $R_{b}<0$ enhance the pressure gradient. Meanwhile, the pseudoplastic impacts $R_{b}>0$ reduce the pressure gradient [8].

The pressure rise $\Delta P$ and the friction force $\Delta F$ are plotted versus the mean flow rate for different values of the Rabinowitsch parameter $R_{b}$ through Figures $7(\mathrm{a})-7(\mathrm{~d})$. Free pumping takes place when $(\triangle P=0)$, the peristaltic pumping occurs for $(\Delta P>0$ and $Q>0)$. However, the retrograde pumping regions arise at $(\Delta P>0$ and $Q<0)$. Meanwhile, the co-pumping occurs for $(\Delta P<0, Q>0)$. It is evident from Figure 6(a) that the peristaltic pumping region enhances with the enhancement of the Rabinowitsch parameter for the pseudoplastic effects $R_{b}>0$.
Meanwhile, it is obvious from Figure 6(b) that the co-pumping region decreases with the boost of the Rabinowitsch parameter for the dilatant effects $R_{b}<0$. Moreover, it is observed that, for the free pumping case, there are no noticeable differences. By contrast, the retrograde pumping region diminishes with the rise of the Rabinowitsch parameter for both the pseudoplastic effects $R_{b}>0$ and the dilatant effects $R_{b}<0$. However, Figures $7(\mathrm{c})$ and $7(\mathrm{~d})$ show that the friction forces have counteractive demeanors when compared with the pressure rise. The relation between the pressure rise and the mean flow rate does not remain linear because of the cubic equation of the Rabinowitsch fluid, which generates a cubic equation of the pressure gradient [8].

4.2. Trapping. Trapping is an essential physical procedure correlated with peristaltic transport. Trapping takes place only in particular circumstances. As shown in the wave frame of reference, a set of closed streamlines can be recognized in the most stretched section of the tube. This set of streamlines is designated as a bolus of fluid. This bolus transmits along with the wave in the laboratory frame. There is an inner circulation which is observed inside the bolus. However, all the involved fluid particles travel with a mean velocity, which is equal to the wave speed. Therefore, the structure of an internal revolving bolus of the fluid is 
designated as a trapping. Additionally, bolus is addressed as a volume of fluid bounded by closed streamlines. The trapping phenomenon has been investigated by many researchers, such as Shapiro [5].

The following figures describe the stream line graphs for various values of maximum height of stenosis and Hall current. Figure 8(a) illustrates the impacts of the maximum height of stenosis $h$ on the trapping. It is revealed that the bolus contracts in size by enlarging $h$. The elevation of the maximum height of stenosis makes the arteries narrower. Therefore, the blood movement streams slowly in the arteries, and this leads to a detraction in the size of the trapping bolus. The impact of the Hall current parameter $m$ on trapping is displayed in Figure 8(a). It is revealed that the trapped bolus increases in size with the rise of the Hall current parameter $m$. In fact, by raising $m$, the effective conductivity impedes. Consequently, it results in a reduction in the magnetic damping force. Therefore, the size of the trapped bolus is enlarged.

\section{Conclusions}

Throughout this work, a theoretical approach is introduced to interpret the impacts of the Hall current and Joule heating on the peristaltic transport of blood flow. The flow streams along a tapered artery in the presence of mild stenosis. The procedure is acted by an external uniform magnetic field. The blood can be determined by the Rabinowitsch model. Furthermore, the Darcy-Forchheimer scheme is imposed via a porous space. Moreover, the impacts of a slip condition on velocity and convective conditions of heat and mass transfer are postulated. In addition, the impacts of radiative heat transfer and chemical reactions are presumed. The combined impacts of viscous dissipation and radiation in energy expression are investigated. Furthermore, the Soret and Dufour features are presumed.

The concluding remarks may be listed as follows:

(1) The axial velocity decreases with the increase of $H^{2}$ because of the increase in the Lorentz force. Also, the axial velocity increases by increasing Hall parameter $m$, and this is due to the decrease in effective conductivity.

(2) The enlarging of the Darcy-Forchheimer number $F_{r}$ enhances the viscous interference and causes a better flow resistance. Therefore, the increase in $F_{r}$ leads to a reduction in the velocity of the flow.

(3) The temperature reduces with the increase of $\beta_{t}$, and this is caused by the reduction of the thermal conductivity of the fluid. Also, the concentration diminishes with the increase of $\beta_{c}$, and this is caused by the reduction of the mass conductivity of the fluid.

(4) The peristaltic pumping region increases with the increase of the Rabinowitsch parameter for the pseudoplastic effects $R_{b}>0$.

(5) The relation between the pressure rise and the mean flow rate does not remain linear because of the cubic equation of the Rabinowitsch fluid that produces a cubic equation of pressure gradient.
Indeed, the current investigation has many applications in many scientific areas such as medicine, medical-industrial, and many other applications. Therefore, it is deemed to be very essential in the field of fluid mechanics. The present study represents the description of blood flow within the arteries.

\section{Data Availability}

No data were used to support this study.

\section{Conflicts of Interest}

The authors declare that they have no conflicts of interest regarding the publication of this study.

\section{References}

[1] B. Rabinowitsch, "Über die Viskosität und Elastizität von Solen (On the viscosity and elasticity of sols)," Zeitschrift für Physikalische Chemie, vol. 145, pp. 1-26, 1929.

[2] S. Wada and H. Hayashi, "Hydrodynamic lubrication of journal bearings by pseudoplastic lubricants (part II, experimental studies)," Bulletin of JSME, vol. 14, no. 96, pp. 279-286, 1971.

[3] U. P. Singh, R. S. Gupta, and V. K. Kapur, "On the steady performance of hydrostatic thrust bearing: Rabinowitsch fluid model," Tribology Transactions, vol. 54, no. 5, pp. 723-729, 2011.

[4] J. C. Burns and T. Parkes, "Peristaltic motion," Journal of Fluid Mechanics, vol. 29, no. 4, pp. 731-743, 1967.

[5] A. H. Shapiro, M. Y. Jaffrin, and S. L. Weinberg, "Peristaltic pumping with long wavelengths at low Reynolds number," Journal of Fluid Mechanics, vol. 37, no. 4, pp. 799-825, 1969.

[6] E. N. Maraj and S. Nadeem, "Application of Rabinowitsch fluid model for the mathematical analysis of peristaltic flow in a curved channel," Zeitschrift für Naturforschung A, vol. 70, no. 7, pp. 513-520, 2015.

[7] Y. C. Fung and F. Yin, "Peristaltic waves in circular cylindrical tubes," Journal of Applied Mechanics, vol. 36, no. 3, pp. 579-587, 1969.

[8] B. K. Singh and U. P. Singh, "Analysis of peristaltic flow in a tube: Rabinowitsch fluid model," International Journal of Fluids Engineering, vol. 6, no. 1, pp. 1717-1722, 2014.

[9] U. P. Singh, "An exact solution of non-Newtonian peristaltic flow in a tube: Rabinowitsch fluid model," Journal of Science of the University of Kelaniya, vol. 10, pp. 1-11, 2015.

[10] K. C. Ang and J. N. Mazumdar, "Mathematical modelling of three-dimensional flow through an asymmetric arterial stenosis," Mathematical and Computer Modelling, vol. 25, no. 1, pp. 19-29, 1997.

[11] W. W. Nichols and M. F. Orourke, McDonald's Blood Flow in Arteries, Oxford University Press, New York, NY, USA, 1973.

[12] N. Verma and R. S. Parihar, "Mathematical model of blood flow through a tapered artery with mild stenosis and hematocrit," Journal of Modern Mathematics and Statistics, vol. 4, no. 1, pp. 38-43, 2010.

[13] N. T. M. El-dabe, G. M. Moatimid, M. A. Hassan, and D. R. Mostapha, "Electrohydrodynamic peristaltic flow of a viscoelastic Oldroyd fluid with a mild stenosis: application of an endoscope," Journal of Applied Mechanics and Technical Physics, vol. 55, no. 4, pp. 565-577, 2016. 
[14] C. P. Arora, Heat and Mass Transfer, Khanna Publishers, Delhi, India, 2nd edition, 1997.

[15] H. Sadaf and S. Nadeem, "Analysis of combined convective and viscous dissipation effects for peristaltic flow of Rabinowitsch fluid model," Journal of Bionic Engineering, vol. 14, no. 1, pp. 182-190, 2017.

[16] N. Ijaz, A. Zeeshan, M. M. Bhatti, and R. Ellahi, "Analytical study on liquid-solid particles interaction in the presence of heat and mass transfer through a wavy channel," Journal of Molecular Liquids, vol. 250, pp. 80-87, 2018.

[17] S. Chakravarty and S. Sen, "Dynamic response of heat and mass transfer in blood flow through stenosed bifurcated arteries," Korea-Australia Rheology Journal, vol. 17, no. 2, pp. 47-62, 2005.

[18] D. Pal and B. Talukdar, "Combined effects of Joule heating and chemical reaction on unsteady magnetohydrodynamic mixed convection of a viscous dissipating fluid over a vertical plate in porous media with thermal radiation," Mathematical and Computer Modelling, vol. 54, no. 11-12, pp. 3016-3036, 2011.

[19] T. Hayat, S. Asghar, A. Tanveer, and A. Alsaedi, "Chemical reaction in peristaltic motion of MHD couple stress fluid in channel with Soret and Dufour effects," Results in Physics, vol. 10, pp. 69-80, 2018.

[20] N. A. Shah, I. L. Animasaun, J. D. Chung, A. Wakif, F. I. Alao, and C. S. K. Raju, "Significance of nanoparticle's radius, heat flux due to concentration gradient, and mass flux due to temperature gradient: the case of Water conveying copper nanoparticles," Scientific Reports, vol. 11, no. 1882, 2021.

[21] E. E. Tzirtzilakis, "A mathematical model for blood flow in magnetic field," Physics of Fluids, vol. 17, no. 7, pp. 077103-077115, 2005.

[22] K. Javid, M. Waqas, Z. Asghar, and A. Ghaffari, "A theoretical analysis of biorheological fluid flowing through a complex wavy convergent channel under porosity and electro-magneto-hydrodynamics effects," Computer Methods and Programs in Biomedicine, vol. 191, Article ID 105413, 2020.

[23] M. S. Iqbal, I. Mustafa, I. Riaz, A. Ghaffari, and W. A. Khan, "Influence of carbon nanotubes on heat transfer in MHD nanofluid flow over a stretchable rotating disk: a numerical study," Heat Transfer, vol. 50, no. 1, pp. 619-637, 2021.

[24] N. T. M. El-dabe and D. R. Mostapha, "MHD peristaltic flow of a Walter's B fluid with mild stenosis through a porous medium in an endoscope," Journal of Porous Media, vol. 22, no. 9, pp. 1109-1130, 2019.

[25] M. M. Bhatti, A. Zeeshan, and R. Ellahi, "Simultaneous effects of coagulation and variable magnetic field on peristaltically induced motion of Jeffrey nanofluid containing gyrotactic microorganism," Microvascular Research, vol. 110, pp. 32-42, 2017.

[26] M. V. Krishna and B. V. Swarnalathamma, "Heat and mass transfer on the peristaltic flow of an incompressible electrically conducting williamson fluid through a porous medium in a symmetric channel with hall current effects and inclined magnetic field," International Journal of Scientific Engineering and Research, vol. 6, no. 9, pp. 941-957, 2015.

[27] Z. Shah, S. Islam, T. Gul, E. Bonyah, and M. Altaf Khan, "The electrical MHD and Hall current impact on micropolar nanofluid flow between rotating parallel plates," Results in Physics, vol. 9, pp. 1201-1214, 2018.

[28] D. Chae, P. Degond, and J.-G. Liu, "Well-posedness for Hallmagnetohydrodynamics," Annales de l'Institut Henri Poincare C, vol. 31, no. 3, pp. 555-565, 2014.
[29] D. Chae and J. Lee, "On the blow-up criterion and small data global existence for the Hall-magnetohydrodynamics," Journal of Differential Equations, vol. 256, no. 11, pp. 3835-3858, 2014.

[30] T. Hayat, S. Nawaz, A. Alsaedi, and M. Rafiq, "Mixed convective peristaltic flow of water based nanofluids with Joule heating and convective boundary conditions," PLoS One, vol. 11, no. 4, pp. 1-28, 2016.

[31] T. Hayat, H. Zahir, A. Alsaedi, and B. Ahmad, "Hall current and Joule heating effects on peristaltic flow of viscous fluid in a rotating channel with convective boundary conditions," Results in Physics, vol. 7, pp. 2831-2836, 2017.

[32] N. T. M. El-dabe and D. R. Mostapha, "Hall Current and Joule Heating effects on peristaltic flow of a sisko fluid with mild stenosis through a porous medium in a tapered artery with slip and convective boundary conditions," Sains Malaysiana, vol. 49, no. 5, pp. 1175-1190, 2020.

[33] H. Darcy, Les Fontaines Publiques de la Ville de Dijon, Hachette Livre Bnf, Paris, France, 1856.

[34] P. H. Forchheimer, "Wasserbewegung Durch boden," Zeitschrift Fur Acker Und Pflanzenbau, vol. 45, pp. 1782-1788, 1901.

[35] A. Shafiq, G. Rasool, H. Alotaibi et al., "Thermally enhanced Darcy-Forchheimer Casson-water/glycerine rotating nanofluid flow with uniform magnetic field," Micromachines, vol. 12 , no. 6 , p. $605,2021$.

[36] M. R. Eid and F. Mabood, "Entropy analysis of a hydromagnetic micropolar dusty carbon NTs-kerosene nanofluid with heat generation: Darcy-Forchheimer scheme," Journal of Thermal Analysis and Calorimetry, vol. 143, no. 3, pp. 2419-2436, 2021.

[37] A. A. Khan, S. Naeem, R. Ellahi, S. M. Sait, and K. Vafai, "Dufour and Soret effects on Darcy-Forchheimer flow of second-grade fluid with the variable magnetic field and thermal conductivity," International Journal of Numerical Methods for Heat and Fluid Flow, vol. 30, no. 9, pp. 4331-4347, 2020.

[38] C.-H. He, D. Tian, G. M. Moatimid, H. F. Salman, and M. H. Zekry, "Hybrid Rayleigh-van der pol-duffing oscillator: stability analysis and controller," Journal of Low Frequency Noise, Vibration and Active Control, 2021.

[39] D. Tian, Q.-T. Ain, N. Anjum, C.-H. He, and B. Cheng, "Fractal N/MEMS: from pull-in instability to pull-in stability," Fractals, vol. 29, no. 2, Article ID 2150030, 2021.

[40] D. Tian and C.-H. He, "A fractal micro-electromechanical system and its pull-in stability," Journal of Low Frequency Noise, Vibration and Active Control, vol. 40, no. 3, pp. 1380-1386, 2021.

[41] K. L. Wang and C. H. He, “A remark on Wang's variational principle,” Fractals, vol. 27, no. 8, Article ID 1950134, 2019.

[42] K. L. Wang, K. J. Wang, and C. H. He, "Physical insight of local fractional calculus and its application to fractional KdVBurgers-Kuramoto equation," Fractals, vol. 27, no. 7, Article ID 1950122, 2019.

[43] X. Li and D. Wang, "Effects of a cavity's fractal boundary on the free front interface of the polymer filling stage," Fractals, Article ID 2150225, 2021.

[44] G.-Q. Feng, "He's frequency formula to fractal undamped Duffing equation," Journal of Low Frequency Noise, Vibration and Active Control, 2021.

[45] S. Chakravarty, A. Datta, and P. K. Mandal, "Analysis of nonlinear blood flow in a stenosed flexible artery," International Journal of Engineering Science, vol. 33, no. 12, pp. 1821-1837, 1995. 
[46] P. K. Mandal, "An unsteady analysis of non-Newtonian blood flow through tapered arteries with a stenosis," International Journal of Non-linear Mechanics, vol. 40, no. 1, pp. 151-164, 2005.

[47] Y. Zuo and H. Liu, "Instability of the printing jet during the three-dimensional-printing process," Journal of Low Frequency Noise, Vibration and Active Control, 2021.

[48] Y. T. Zuo, "Effect of SiC particles on viscosity of 3-D print paste: a fractal rheological model and experimental verification," Thermal Science, vol. 25, no. 3B, pp. 2405-2409, 2021.

[49] Y. H. Liang and K. J. Wang, "A new fractal viscoelastic element promise and applications to Maxwell-rheological model," Thermal Science, vol. 25, no. 2, pp. 1221-1227, 2021.

[50] J.H. He, "Seeing with a single scale is always unbelieving: from magic to two-scale fractal," Thermal Science, vol. 25, no. 2, pp. 1217-1219, 2021.

[51] N. Anjum, C. H. He, and J. H. He, "Two-scale fractal theory for the population dynamics," Fractals, 2021.

[52] W. M. Rohsenow, J. P. Hartnett, and Y. I. Cho, Handbook of Heat Transfer, McGraw-Hill, New York, NY, USA, 1998.

[53] J.-H. He, "Homotopy perturbation technique," Computer Methods in Applied Mechanics and Engineering, vol. 178, no. 3-4, pp. 257-262, 1999.

[54] S. Nadeem and N. S. Akbar, "Peristaltic flow of Sisko fluid in a uniform inclined tube," Acta Mechanica Sinica, vol. 26, no. 5, pp. 675-683, 2010.

[55] A. M. Siddiqui, Q. A. Azim, A. Ashraf, and Q. K. Ghori, Topological Methods in Nonlinear Analysis, vol. 31, no. 2, pp. 331-339, 2008.

[56] T. Hayat, M. Rafiq, and B. Ahmad, "Soret and Dufour effects on MHD peristaltic flow of Jeffrey fluid in a rotating system with porous medium," PLoS One, vol. 11, no. 1, Article ID e0145525, 2016. 